\title{
Cellular Automaton experiments on local galactic structure. I. Model assumptions
}

\author{
J. Perdang ${ }^{1}$ and A. Lejeune ${ }^{2}$ \\ 1 Institute of Astronomy, Madingley Road, Cambridge CB3 OHA, UK, and Institut d'Astrophysique, 5, Avenue de \\ Cointe, B-4000 Liège, Belgium* \\ 2 Institut de Physique, Sart-Tilman, B-4000, Liège, Belgium
}

Received October 27, 1994; accepted March 6, 1996

\begin{abstract}
The purpose of the present paper, combined with the companion paper (Lejeune \& Perdang 1995, hereinafter Paper II), is to demonstrate that a Cellular Automaton (CA) framework incorporating detailed physical evolutionary mechanisms of the galactic components provides a straightforward approach for simulating local structural features in galaxies (such as those of flocculent spiral galaxies). Conversely, and more important, the observed local irregularities may give information on the relevant timescales of the evolutionary processes operating in these galaxies. In this paper we start out with a critical review of the more standard methods in use in galactic modelling. We insist on the fact that these models do not lend themselves to a straightforward inclusion of both the galactic dynamics and the physical evolution of the galactic components. We show that the Cellular Automaton approach can combine both effects, on condition that the dynamics is approximated by a stationary, in general space-dependent velocity field of the galactic matter. The main part of the paper addresses an extension of the Stochastic Propagating Star Formation scheme originally devised by Mueller \& Arnett (1976). The model consists in a multi-state 2D CA specifically designed to deal with the evolutionary behaviour of an off-centre region of a galaxy, of an area of a few $\mathrm{kpc}^{2}$. The model incorporates a detailed sequence of in part parametrised stellar evolutionary processes. In the version discussed here it includes as dynamical effects the motions of galactic matter due to a stationary circulation and, to some extent, due to the proper motions of the stars. The model we present is a first nontrivial instance of a CA defined over a lattice lacking geometric symmetries (crystal symmetries of standard CA, or rotational symmetry of the Mueller-Arnett CA). The precise geometry of the CA network of cells is imposed in our model by the space-dependent stationary galactic velocity field. Numerical results are discussed in the companion paper.
\end{abstract}

Key words: galaxies: structure — star formation — methods: numerical

\section{Introduction}

From the point of view of the formal theory of dynamical systems (DS), current galactic models belong into three broad categories.

\subsection{Low order differential models}

On the one end of the spectrum of models, we have a class of elementary DSs, namely ordinary differential equations (ODEs) of low order (say $n=3,4, \ldots$ ). Such models are adopted to supply a theoretical basis for an understanding of the gross evolutionary behaviour of the local stellar content, together with the chemical evolution of the galaxy. The formulation, dealing with a local part of the galaxy only, is reminiscent of chemical kinetics in a homoge-

Send offprint requests to: J. Perdang

${ }^{\star}$ Permanent address neous medium (Kaufman 1979; Shore 1981, 1982; Cowie \& Rybicki 1982; Franco \& Shore 1984; Shore et al. 1987; cf. also comments in Shore 1985). The counterparts of the chemical species are here the galactic species (interstellar gas and dust, and different classes of stars); the rates of the transformation processes of one species into another typically involve adjustable parameters. The main virtue of these models is that they demonstrate the important role of nonlinear steps in the evolution equations: Through nonlinear feedback the evolution may exhibit generically oscillatory as well as more complex stationary fluctuations, while a purely linear dynamics would imply an asymptotic evolution towards a time-independent state. Being of low order, the ODE formulations lend themselves to a more detailed mathematical analysis (Shore 1985). For $n \geq 2$ these models have parameter ranges over which the evolutionary behaviour is periodic; for $n \geq 3$ they may in principle exhibit deterministic chaos over some range, 
although such a behaviour has not yet been reported so far. By ignoring spatial exchanges - diffusion in the analogue of chemical kinetics - such models automatically discard, to a large extent, the details of the interactions between different species.

In spite of their simplicity, these models may also produce nontrivial space patterns: If the local transformation rates include a slight systematic radial space dependence, then in a periodic regime the ensuing space dependence of the period is responsible for the formation of annular isodensity lines of the different constituents (cf. Perdang 1974); the kinematics of rotation is then sufficient to deform the latter into spirals. ${ }^{1}$

Within this class of models global mechanical effects, such as galactic rotation and gravitation, are entirely ignored.

\subsection{High order differential models}

At the other end of the spectrum of galactic models we have a class of DSs in the form of ODEs of high or$\operatorname{der}\left(>10^{3}\right)$, namely $N$-body simulations. Following the initial attempts of the late 60's (Miller \& Prendergast 1968; Hockney \& Hohl 1969; cf. also Aarseth 1972), these originally $2 D$ models incorporate as only physics gravitation (combined with rotation through appropriate initial conditions). In principle, these models cope therefore with the correct global galactic mechanics, though even the most accurate $N$-body programs, handling some $10^{6}-10^{7}$ bodies, work at a numerical 'noise level ... about one thousand times higher than ... a galaxy' (Sellwood \& Wilkinson 1993). $N$-body calculations generate spatial inhomogeneities: With simplest initial conditions for a galaxy (statistically uniform density; systematic rotation with superposed random motions of the individual bodies; absence of a halo), these experiments produce a (transient) double arm connected by a central bar (cf. the original $2 D$ experiments by Hohl 1971), or more generally, coherent spatial matter concentrations reminiscent of spiral arms and bars (cf. the $3 D$ experiments by Raha et al. 1991; and the analysis by Pfenniger \& Friedli 1991).

$N$-body models are predicated on the assumption that the constituents are structureless permanent point masses; the intrinsic physical evolution of the individual galactic species is ignored. On the other hand, the prominent global observational structures in a galaxy, such as the geometry of a spiral arm or of a central bar, are of an 'optical' nature. The observed geometry is the geometry of the isophotes; any photometric or photographic technique supplies a brightness map, rather than a direct picture

\footnotetext{
${ }^{1}$ If diffusion is superimposed on the chemical kinetic equations - transforming the elementary DS into a different class of a DS, namely a system of partial differential equations (PDEs) - , the oscillating regime gives rise to travelling waves which again can form spiral patterns (Cowie \& Rybicki 1982; Feitzinger 1985).
}

of hydromechanical properties (such as the distribution of matter density). While the statement " 'brightness' requires the 'presence of matter' ", is manifestly true, the converse statement " 'presence of matter' implies 'optical observability' ", does not hold. Any inferences on the matter distribution from optical observations (including radio, $\mathrm{cm}, \mathrm{mm}, \mathrm{IR}, \mathrm{UV}, \mathrm{X}$ and gamma) are indirect, and necessarily based on disputable, and disputed, assumptions on the chemical nature and the physical state of the galactic matter.

High luminosity in optical light, on the other hand, is chiefly the result of the presence of stars in their earliest evolutionary phases. It is then not obvious to conceive that purely mechanical $N$-body models, interesting as they may be, should satisfactorily account for an interpretation of the salient brightness patterns of galaxies as shown for instance in blue light photographs.

\subsubsection{The problem of reduction of the order}

On the mathematical side, the high order $n(=6 N$ in $3 D$ experiments) of the ODEs representative for $N$-body models constitutes a major hurdle to a theoretical investigation of the allowed time and associated space behaviour of these DSs. Accordingly our knowledge of the dynamics of these models mainly rests on numerical experimentation, even though ad hoc approximation schemes may provide clues towards interpreting the results. Thus the substitution to the instantaneous $N$-body potential of a smoothed out stationary potential decouples the masspoints, and lends itself to an analysis and classification of the allowed orbits (cf. Binney \& Tremaine 1987 Chapt. 3; cf. de Zeeuw \& Franx 1991 for comments on models for elliptical galaxies); in this context, the observation of bars in the $N$-body experiments may be attributed to the existence of 'box orbits' in the mean potential approximation. The method devised by Schwarzschild (1979, 1982 ) is a refinement of this approach, in which a selfconsistent smoothed stationary potential is numerically generated from a large collection of trial orbits, via an iterative technique. A more pragmatic method is adopted by Contopoulos and coworkers (Patsis et al. 1991); these authors relate the smoothed stationary potential (with superposed spiral perturbation) to a parametrised analytical interpolation formula of the rotation curve of a galaxy; the free parameters are then observationally fitted; the density structure of the galaxy is finally simulated using a large enough sample of computed orbits.

Models of this type have been successful in reproducing the spatial distribution of the molecular gas (simulated as test particles; cf. for instance Sempere et al. 1995 in the case of M100).

It is true that very often dynamical systems with large numbers of degrees of freedom asymptotically relax on a short timescale to an internal equilibrium in which a 
substantial fraction of the actual degrees of freedom cease to participate in the dynamics. ${ }^{2}$

Under those conditions, once an internal equilibrium is established, the physically relevant evolution depends effectively on the small number of order parameters alone, which in turn obey the dynamics of a low order ODE. A mean potential representation, to the extent that it is an asymptotically correct description of the $N$-body behaviour, may perhaps be justified on the basis of this general observation.

A systematic, and mathematically precise method that achieves the reduction of degrees of freedom is the Poincaré Normal Form technique (cf. Arnold 1976). This technique demonstrates that a reduction to a low order of the originally high order ODEs representative for the numerical treatment of the dynamics of a star is not allowed in the general $3 D$ case (while such a reduction does hold if the dynamics is forced to be strictly radial): in the $3 D$ case the reduced ODE remains of a very high order (Perdang 1994). Due to a formal similarity of the equations of stellar and galactic dynamics, we may infer that the stellar non-reducibility should extend to the case of galactic structure. Simplified models, such as the mean potential approach, may supply partial information on limited sub-problems; to understand the general aspects of the $N$-body dynamics, however, actual large scale numerical experiments appear as inevitable.

\subsubsection{The fluid dynamic approach}

A macroscopic variant of the microscopic $N$-body equations is a formalism of fluid dynamics as derived from a Boltzmann (Vlasov) equation approach. The formulation of fluid dynamics belongs into a new category of DSs, namely partial differential equations (PDEs). Although relying on a spatial smoothing of the microscopic dynamics, the macroscopic PDE formulation preserves the large (formally infinite) number of degrees of freedom. It is therefore not subject to the above criticism and can serve the purpose of interpreting the global behaviour of $N$-body experiments.

Linear stability calculations of differentially rotating discs initiated by Lin \& Shu (1964, 1966; also Lin 1966) in the context of this model demonstrate the existence of unstable modes. The density pattern of the most unstable mode, reminiscent of the observed structure of spiral arms, is then identified with the global spiral structure - the Grand Design of $\mathrm{Sa}, \mathrm{Sb}$ and Sc galaxies - (cf. Shu 1991 Chapt. 11; Bertin et al. 1989; cf. also Binney \& Tremaine 1987 chapt. 6) as well as with the matter

\footnotetext{
${ }^{2}$ More precisely, in the terminology of Haken, a large number of the actual degrees of freedom are 'slaved' by a few relevant degrees of freedom, or 'order parameters' (Haken 1977 chapt. 7). The latter collective degrees of freedom alone eventually govern the macroscopic time behaviour.
}

concentrations as traced in the $N$-body experiments; this conjecture is the main ingredient of the density wave theory. Although actual calculations are limited to the linear regime, a physical argument (nonlinear stabilisation of the linearly unstable mode as a result of dissipation leading to a stationary finite amplitude pattern; Kalnajs 1972) suggests that the global pattern is the outcome of a single symmetry-breaking in the uniform disc induced by a dynamic instability. ${ }^{3}$

Theoretically it is not obvious why the geometrically smooth linearly unstable pattern should preserve its incipient shape in a nonlinear regime. Nothing excludes a priori the occurrence of a hierarchy of superposed symmetry breakings away from the regular stationary arm pattern; this hierarchical scenario would in turn lead to increasingly less trivial stationary as well as nonstationary geometries. We recall in this connection that CouetteTaylor flow, not unrelated to the large scale flow set up in a rotating galaxy, is indeed subject to a variety of symmetry-breaking instabilities, as is known both theoretically and experimentally (Brandstadter \& Swinney 1987; Cross \& Hohenberg 1993). Likewise, the geometry of a hydrodynamic vortex exhibits an irregular spiral pattern reminiscent of spiral galaxies (cf. the vortex simulations in Lavallée et al. 1993).

Finally, even in the case of the geometrically most regular galaxies the observed structure of the galactic arms does not really exhibit the smooth and regular shape of the unstable linear density waves (cf. M81 chosen in King 1989a to demonstrate the Grand Design; cf. in particular the plates pp. 99-105, 119-127 in the Revised ShapleyAmes Catalog, Sandage \& Tamman 1981 (RSAC), and the large scale plates (Part II) in the Atlas of Galaxies, Sandage \& Bedke 1988 (AG)).

Besides these weaknesses proper to the linear density wave theory, any fluid dynamic approach - or nonlinear wave theory - shares what we believe is the major weakness of the $N$-body model which it is meant to reproduce: Like the latter, it ignores any evolutionary effect in the individual galactic species. On the other hand, the fluid dynamic approach may remain meaningful for dealing with the interstellar gas. Regarding the interstellar medium, the atomic component is typically observed via $\mathrm{HI}$ in the Milky Way (since 1951, $21 \mathrm{~cm}$ line); as a tracer of the molecular component, in particular of the giant molecular clouds (GMC), and hence of the regions of star formation, CO is used (1.3 and $2.6 \mathrm{~mm}$ line; cf. King

\footnotetext{
${ }^{3} \mathrm{~A}$ symmetry-breaking from an original axisymmetry $\left(C_{\infty}\right.$ group) necessarily consists in a transition to a subgroup of the latter, i.e. to some $C_{m} \subset C_{\infty}$ ( $m$-fold rotational symmetry), regardless of the physics involved; representatives of $C_{m}$ are $m$-fold arms. The specific selection $m=2$ as already exhibited in Hohl's (1971) $N$-body experiments - and in real galaxies - depends on the precise physics responsible for the instability.
} 
1989; Combes 1991). As is convincingly demonstrated by a comparison of a plot of the HI column density brightness in M81 (Fig. 6-23 in Binney \& Tremaine 1987) with a blue-light photograph of this galaxy (p. 123 in the RSAC), the HI distribution globally follows the arms of the photograph, even though the two specific geometries are not identical (see also Fig. 10.8 in van der Kruit 1989, showing a similar situation for NGC 628). Maps of the CO distribution in M51 compared with various other maps ( $\mathrm{HI}, \mathrm{H} \alpha$ ) exhibit elongated patches sketching the global shapes of ragged arms; the arm structures of $\mathrm{CO}, \mathrm{HI}$ and $\mathrm{H} \alpha$ are again roughly, though not exactly, coincident (cf. Fig. 3 in Young \& Scoville 1991).

\subsection{Cellular Automaton models}

As a category of galactic structure DSs not encoded in conventional ODEs or PDEs, a novel methodology was introduced in the $70 \mathrm{~s}$, in the form of cellular automaton models. With a discretised time, a discretised space $(2 D$ in the majority of models devised so far), and with a discrete representation of the physics, the dynamics of the CA is captured algebraically by an iteration in the integral domain of the integers (a system of a large number $\left[>10^{5}\right]$ of difference equations in integer variables). The latter can therefore be solved exactly. This is a major computational advantage of a CA model over ODE and PDE formulations whose numerical solutions are always subject to round-off errors: Instabilities, especially multiple competing instabilities, are hard to control in an ODE or PDE framework where round-off errors are then amplified exponentially in time. Since, as stressed above, such instabilities are not unlikely to occur in real galaxies, we believe that the CA environment is a computationally more satisfying framework for galactic modelling.

As a further advantage of the CA framework for handling physics, we point out that physical mechanisms hard to translate into ODE or PDE form are often trivially expressed as CA rules (cf. the discussion in Perdang 1993). ${ }^{4}$

\footnotetext{
${ }^{4} \mathrm{An}$ illustration is the chaotic mechanism for the formation of irregular structure as suggested by Goldreich \& LyndenBell (1965) and which so far remained on a descriptive level. According to Elmegreen \& Elmegreen (1982) about $70 \%$ of the isolated spiral galaxies are flocculent; they exhibit 'short, chaotic-looking arms and no apparent symmetry' (Elmegreen \& Elmegreen 1983). To account for the raggedness and patchiness of real galactic arms Goldreich and Lynden-Bell propose the following scenario: Through a spontaneous Jeans instability in a clump at some random location in the galaxy and at some random instant of time, a nest of protostars is created. The kinematic shearing effect of the differential rotation subsequently stretches this aggregate of young objects into an elongated, irregular patch - a local portion of a luminous arm. Due to the short lifetime of the high luminosity phase of the young stars, the actual visibility of such a localised arm structure is severely limited in time. An impression of a global continuous
}

The first attempt at simulating the global morphology of spiral galaxies in the framework of a CA model was due to Mueller \& Arnett (MA) (1976). The CA formulation was carried further in a series of papers by Gerola, Seiden, Schulman and coworkers (GSS) (Gerola \& Seiden 1978; Seiden et al. 1979, 1982; Seiden \& Gerola 1979; Gerola et al. 1980; Schulman \& Seiden 1982; for discussions and extended reviews see Seiden \& Gerola 1982; Schulman \& Seiden 1983, 1986; Seiden \& Schulman 1990; Schulman 1993), as well as by Comins \& coworkers (C) (Comins 1981, 1983, 1984; Statler et al. 1983; Balser \& Comins 1988; Comins \& Shore 1990). Essentially, a galactic CA model may incorporate any physical ingredient of the low-dimensional ODE models listed under (1), with an additional implementation of space dependence.

The simplest CA version is based on 4 physical assumptions and approximations:

(a) The model incorporates two galactic species only, namely 'gas' or 'dust', $D$, and 'stars', $S$ (in the form of luminous high mass stars); each cell of the automaton is either in the $D$ state, or in the $S$ state.

(b) The model takes account of the kinetics of the observationally documented processes of star formation in a parametrised form. These mechanisms are:

$(\alpha)$ spontaneous star formation, viewed as any process conforming to a decay reaction

$$
D \rightarrow S
$$

a cell in state $D$ spontaneously transforms into a state $S$ with a given probability per timestep (simulation of a spontaneous Jeans instability of the interstellar medium); and

( $\beta$ ) star-induced star formation, or self-propagating star-formation (SPSF) in the terminology of MA; formally SPSF refers to the totality of autocatalytic processes obeying a stoichiometric scheme

$$
D+m S \rightarrow(1+m) S
$$

a cell in state $D$ transforms into a state $S$ with a given probability per timestep provided that there are $m$ cells in state $S$ in the neighbourhood. This step accounts for the observational evidence of star formation (presence of OB associations) in expanding spherical shells surrounding supernovae and high luminosity stars in general (Sancisi 1973; Berkhuijsen 1974; Castor et al. 1975; Elmegreen \& Lada 1977); these observations are interpreted as the effects of shocks generated by supernova explosions (Arnett et al. 1989), shocks associated with $\mathrm{H}$-ionisation fronts

spiral arm pattern is eventually created by the simultaneous presence of a large number of such short-lived elongated luminous patches appearing and disappearing at random positions; the overall shape of the arm reflects the direction of the stretching. It is not clear how this mechanism could be captured by a PDE model; on the other hand the CA framework described below incorporates the mechanism automatically. 
due to OB stars (Elmegreen \& Lada 1977, who quote M42, M17, M8, NGC 7538), or shocks connected with stellar winds generated by massive stars. These shocks compress the interstellar gas into molecular clouds, or the clumps in a cloud, which in turn transform into stars. ${ }^{5}$

(c) The standard CA model incorporates a stationary circular differential rotation of the galaxy (cells are arranged in rings which are rotated at different speeds, in accordance with a given rotation law). Rotation thus plays a kinematic role only, thereby influencing however the processes of star formation. Gravitation is not explicitly handled in this CA version; this should not be equated with saying that gravitation is just ignored. The stationary kinematic motion which is implemented is to be interpreted dynamically as resulting from all forces - including gravitation - acting on the galactic matter. Although an improvement over the ODE models (1), assumption (c) corresponds to a lower order approximation of the galactic dynamics than the mean potential method mentioned under (2): Stationary rotation amounts to taking account of the simplest class of dynamically allowed orbits only, namely circular orbits in a mean radial gravitational field; moreover, the possible occurrence of resonances - and the possibility of chaotic dynamics - is ignored. The averaged potential models under (2) sample in principle all categories of allowed orbits including chaotic dynamical behaviour.

(d) Initially all cells are in the ('active') $D$ state.

If spatial structure emerges in this model, then it is not due to a direct action of gravitation on matter; this contrasts with models (2), in which the wave pattern is related exclusively to the inhomogeneities in the matter density. In a CA model obeying the kinematic assumption (c) the originally uniform overall density distribution (assumption (d)) is preserved; structure can only arise as a consequence of the evolution of the galactic components (asumption (b), Eqs. (1.1) and (1.2)); the global motion and hence indirectly gravitation modulate however the local rates of these evolutionary processes. The develop-

\footnotetext{
${ }^{5}$ In the context of the CA models of GSS the simulation parametrises the rates of the 'chemical reactions' (1.1) and (1.2). To the extent that the star formation mechanisms conform to these reactions, the simulation remains invariant under any specific interpretation of the two mechanisms. It is irrelevant whether it is a real shock or any other physical process (for instance a gravitational interaction, or some other energy transfer) that induces the transformation of gas into stars in process (1.2), provided only that this process requires the presence of nearby stars. In GSS the gas component $D$ is subdivided into an 'active' and an 'inactive' phase parametrised by a 'refractory time' $\tau$. Essentially, if a star formation event has occurred in a given cell at a given time, then during $\tau$ timesteps this same cell remains 'inactive' in the sense that if it contains gas then the latter is not allowed to transform into stars. We observe in passing that the refractory time is a parameter which is hard to estimate physically.
}

ment of a pattern corresponds to a spatial organisation of the two galactic components, rather than to a global rearrangement of matter density (as happens in models $(2))$.

It was recognised by MA that it was the specific SPSF mechanism - the autocatalytic step (1.2), i.e. the presence of a nonlinear fragment in the 'reaction kinetics' which was eventually responsible for the development of a chain of contiguous active cells interpreted as the luminous galactic arm. Schulman and coworkers subsequently identified the formation of the arm pattern with a percolation phenomenon in the star population (modified by the galactic kinematics; cf. Schulman \& Seiden 1983, and Seiden \& Schulman 1990 for analytical details). If all model parameters except the probability of induced star formation, $p$ (process 1.2), are kept fixed, then the correlation length of the star population, $\xi(p)$, and the mean cluster size of the star population (total number of stars in a group of stars occupying contiguous cells), $S(p)$, diverge as

$\xi(p) \sim\left|p_{\mathrm{c}}-p\right|^{-\nu}(a)$, and $S(p) \sim\left|p_{\mathrm{c}}-p\right|^{-\gamma}(b)$,

as the free parameter $p$ approaches a critical value $p_{\mathrm{c}}$ (cf. Stauffer 1985). Given the geometry of the cellular grid of the CA, the critical exponents $\nu, \gamma(>0)$ can be estimated theoretically. For a probability $p<p_{\mathrm{c}}$ the catalytic process (1.2) remains too inefficient to secure the actual propagation of star formation; there will be no unbounded cluster of activity. A huge cluster of stellar activity - a galactic arm - is formed at the percolation phase transition when $p$ tends to $p_{\mathrm{c}}(S(p) \rightarrow \infty){ }^{6}$

Even these simplest CA schemes have been surprisingly successful in generating synthethic pictures of galaxies which closely resemble photographs of real galaxies (cf. the comparisons of CA simulations with the blue plates of NGC 628 [Sc(s)I in the classification scheme of the RSAC] and NGC 7793 [Sd(s)IV] in Schulman \& Seiden 1986, or Seiden \& Schulman 1990). Photographs of real galaxies are in fact far better mimicked by CA simulations than by the computationally much more involved full $N$-body simulations (cf. for instance Sellwood \& Carlberg 1984) or approximate many-body simulations in given gravitational fields (possibly modified by self-gravitational effects; cf. the simulations of molecular cloud distributions in Sempere et al. 1995). Whatever the critique directed

\footnotetext{
${ }^{6}$ To secure the actual realisation of a proper galactic arm pattern a further condition must be satisfied. If the reaction probability exceeds a second critical value $p_{\mathrm{M}}\left(>p_{\mathrm{c}}\right)$ then essentially all matter is in the active star state and the configuration is uniformly luminous; there are no individual arms (cf. in particular the survey in C 1981). Accordingly, in order to guarantee the formation of a realistic arm pattern, the probability parameter $p$ must be confined to some interval $\left(p_{\mathrm{c}}, p_{\mathrm{M}}\right)$.
} 
against the admittedly elementary, and incomplete, nature of this CA model, its success in duplicating real photographs of galaxies secures that it deserves credit, at least as a tool of heuristic value and efficacy.

Reviews of spiral structure theory characteristically present the density wave scheme and the SPSF CA as two rival theories designed to interpret the formation and the stability of galactic arms (cf. Shu 1985). In the former model gravitation coupled with rotation is responsible for a mechanical (second order) phase transition from spatial homogeneity to spatial structure in the global matter distribution. In the latter model, the change in the morphology leaves the global matter distribution essentially unaffected; structure results from inhomogeneities in the space distribution of the galactic components created in a percolation-like phase transition caused by the SPSF mechanism, and modulated by the galactic mechanics.

Since both dynamics and catalytic star formation processes coexist in real galaxies, the two instability mechanisms, rather than being antagonistic, are complementary, and they may well be operative simultaneously. This belief was already expressed by MA who suggested that the SPSF effect might be responsible for the more 'irregular optical appearance often seen in late-type spiral galaxies', while the classic symmetric two-arm spirals might be of gravo-rotational origin. The same idea has been repeated more recently by Schulman (1993, p. 312) who argues that 'some [galaxies] are dominated by gravitational and fluid dynamic effects, while for others the morphology is a consequence of the statistical mechanics effects' as captured in the CA model.

If it is true that the morphology is at least to some extent determined by the SPSF effect, and, more generally, by evolutionary effects of the galactic components (gas, different species of stars, ...), then we are entitled to expect that in turn the morphology may supply information on the evolutionary processes of these components. It is this 'inverse problem' which motivates our interest in concentrating on a more detailed physical model of the internal evolution.

\section{The adopted methodology}

In view of the above remarks, a satisfactory galactic model should incorporate both instability mechanisms, the dynamical density wave effect and the SPSF process. In principle, this could be achieved either in the context of models of category (2), by superposing stellar formation and evolution on an already time-consuming mechanical $N$-body problem (or on the averaged potential variant thereof); or, in the framework of models of category (3), by explicitly handling the galactic dynamics within a CA model. Unfortunately, neither procedure is easy to implement.

On the other hand, in a simulation of galaxies in which the flocculent character is well developed, we believe that the global mechanical effects play a relatively minor part in determining the structure (cf. the comments of MA), so that we are entitled to treat galactic mechanics as a 'perturbation'. We then propose the following hybrid method which takes account of the specific advantages of both approaches (2) and (3):

(a) The dynamics, in the form of the $N$-body problem (2), (or of a convenient approximation to the latter; cf. the mean stationary potential approximation), is solved separately; a hydrodynamic velocity field $\mathbf{v}(\mathbf{r}, t)$ is then derived by ensemble-averaging. Provided that the generally accepted assumption of stationarity holds, the velocity field $\mathbf{v}(\mathbf{r})$ may directly be determined observationally (cf. Fig. 6-23 in Binney \& Tremaine 1987 showing $\mathbf{v}(\mathbf{r})$ in M81 as estimated from the HI distribution). The latter approach was in fact followed by the first proponents of the SPSF model, with the further simplifying assumption that the stationary velocity field is purely rotational.

(b) The CA model is then run with the velocity field $\mathbf{v}(\mathbf{r}, t)$ (or $\mathbf{v}(\mathbf{r})$ ) as given in step (a) added as a kinematic constraint. Under the theoretical alternative of (a), the sequence of steps (a) and (b) may be repeated until convergence.

A flexible technique for implementing an arbitrary space-dependent stationary velocity field in a CA environment consists in adopting a network of cells whose shapes and sizes are functions of position determined by the velocity field itself (cf. the discussion and illustration of this procedure in Perdang 1993); the specific details of this technique are discussed below. The method in the form adopted here appears to be a convenient strategy for a direct simulation of small parts of a galaxy. To model a whole galaxy, a variant of it which remains closer to the standard CA implementation of a pure rotational velocity field as adopted by previous authors (MA, GSS, C) is probably more convenient (cf. below).

Besides our concern for improving the treatment of the galactic dynamics in the CA modelling environment, the present work is motivated by several further observational and theoretical points.

The large-scale plates of spiral galaxies in the AG demonstrate that the geometry of the spiral pattern of even the most regular grand-design galaxies is not fully consistent with the geometrically smooth and regular shape of unstable density modes (cf. Binney \& Tremaine 1987, Chapt. 6.3). The observed patterns are always ragged and fragmented, exhibiting alternating high and low brightness concentrations along an arm. The clearcut geometry of a theoretical linear mode transpires only after strongly smoothing and filtering the galaxy pictures. On the other hand, the actually observable small-scale structure of the isophotes bears a visual similarity with the isophotes of molecular clouds, suggesting in turn that physically similar mechanisms may be operative on the scale of the arms and on the scale of the clouds. Bazell \& Désert (1988) who analysed the geometry of constant 
brightness contours of several molecular clouds (IRAS Sky Flux plates) reported fractal dimensions in the range 1.12-1.40 for the latter; a morphological image analysis of the $\eta$ Carinae cloud (ESO plate) by Blacher \& Perdang (1990) led to fractal dimensions of different brightness levels in the range 1.33-1.45. We expect therefore that the contours of the arm structure are characterised by fractal dimensions in a similar range, say 1.1-1.5.

Density wave theory in its elementary linear form is not consistent with fractality of the arms. In a nonlinear variant, density wave simulations based on averaged potentials do generate fractured irregular arms (especially near the 4/1 resonance; cf. the simulated photograph Fig. $8 \mathrm{~b}$ in Patsis et al. 1991); however, a mere visual comparison of the shape of the simulated arms (cf. again Fig. 8b in Patsis et al. 1991) with the arms of real galaxies indicates morphological differences which are hard to quantify; moreover, it remains to be shown that the conjectured fractality of the simulations is not just an artifact of the low number of sampling orbits. In the framework of gravity-induced dynamics a full existence proof of a transition to fractality is lacking, although numerical experiments are not inconsistent with an evolution towards a hierarchical gravitational organisation (cf. for instance the $1 D$ experiments in Rouet et al. 1991). ${ }^{7}$

In contrast, it is well known experimentally that in CA models fractal structures are common, and for a variety of simple CA evolution rules the occurrence of fractal (space or space-time) structures can be proved analytically (cf. Perdang 1993). We also recall that percolation clusters have fractal boundaries. Accordingly, a CA formulation seems to be an adequate framework for a study of the formation of a fractal arm morphology.

Besides a fractal geometry of the arm structure, a second type of spatial fractality is to be expected: It has been suggested (Ikeuchi \& Yoshioka 1989) that the supernova remnants are fractally distributed in space, in the sense that these objects uniformly cover a fractal region of the galaxy. Since stellar evolutionary effects are not easily implemented in models of class (2), we adopt a CA formalism which can accommodate several stellar species without undue computational complications. In the context of CA models it is a well known experimental fact that specific cellstates can obey fractal space distributions; moreover, for some simple CA evolution rules the existence of frac-

\footnotetext{
${ }^{7}$ The special equations of isothermal self-gravitating $2 D$ fluid dynamics of radial symmetry (matter in an infinite circular cylinder), containing an ad hoc modification to deal with a possible fractal matter distribution, can be shown to be invariant under a Lie group acting also on the dimension of the matter distribution; physically this result means that the system may be deformed without energy expenditure, and hence it may spontaneously evolve from an original non-fractal geometry - the standard matter distribution in the cylinder to a fractal geometry (Perdang 1995).
}

tal distributions can be shown analytically (cf. Perdang 1993).

\section{Geometry of the CA simulation}

In contrast with the CA models as designed by MA, GSS and $\mathrm{C}$, which deal with a global simulation of an entire galaxy, the model we introduce here addresses the morphology of a small part of a galaxy only. It aims at simulating irregular luminous matter concentrations (irregular arm structures) in off-centre regions of intermediate type galaxies (between extreme flocculent and grand-design). Specimens of such galaxies are M33 (arm class 5), or M100 and M101 (arm class 9) (Elmegreen 1981).

An instance of a typical structure we attempt to mimic is provided in Fig. 1 which exhibits an enhanced-contrast digitised picture of an area of about $4 \times 4 \mathrm{kpc}^{2}$ of a real plate of the Sc(s) I type galaxy M101 (NGC 5457). ${ }^{8}$

The schematic morphology shows a conspicuous luminous patch - a ragged arm - extending from the lower left to the upper right corner; the patch appears as subdivided into a number of fragments of various widths (about $0.7 \mathrm{kpc}$ towards the left, $<0.5 \mathrm{kpc}$ towards the centre; on the right we have a broader bright spot). Towards the lower right a further broad elongated bright area is visible, while the upper left area appears to be virtually empty. Figure 1 also shows the results of various image transformations (Gaussian blurring, and contrast enhancement) carried out on the original photograph. These transformations change the apparent linear sizes of the visible structures; they even alter the connectivity of the luminous branches, thereby enhancing or deteriorating structural details. The overall morphology, however, remains unaltered: The broad morphological features, namely brightness concentrations along privileged directions and irregular fragmented nature of the bright areas, are independent of the particular transformation of the original photograph.

All CA simulations we shall be dealing with concern $2 D$ galactic regions of sizes comparable with the area of Fig. 1. Our purpose is to model a local galactic geometry consistent with the broad morphological features of this figure.

On a quantitative side the simulation is eventually intended to serve the purpose of a translation device of selected observational data on the geometry (spatial distribution of galactic components, geometrical structure of spatial support of galactic components) and on the physical composition (mass fractions of different galactic components) into quantitative information about the various

\footnotetext{
${ }^{8}$ Distance of M101: $7 \mathrm{Mpc}$ (AG); scale of $120^{\prime \prime}$ marked out on AG photograph corresponds to $4.1 \mathrm{kpc}$; ratio of principal axes $a / b=1.02$; centre of picture at $6 \mathrm{kpc}$ from centre of galaxy; radius at surface brightness level $25 \mathrm{mag} / \operatorname{arcsec}^{2}, R_{25}=$ $28.2 \mathrm{kpc}$ (Elmegreen \& Elmegreen 1982).
} 
(a)
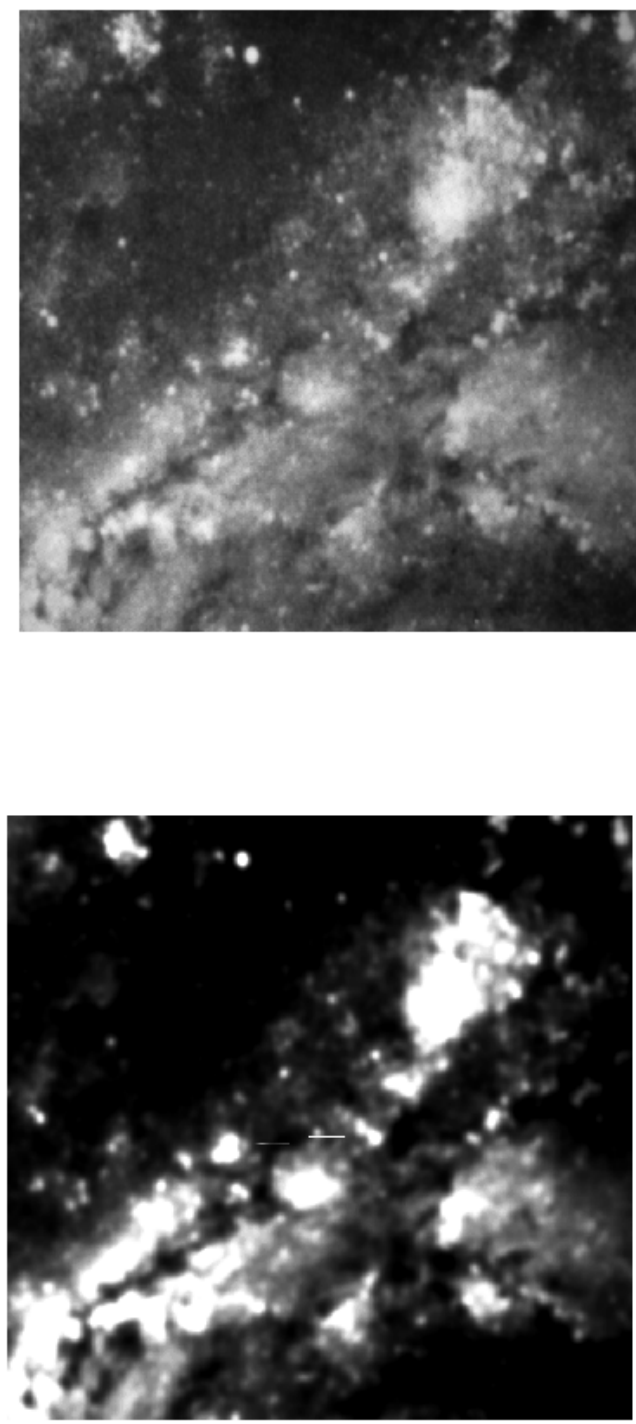

(c) (b)
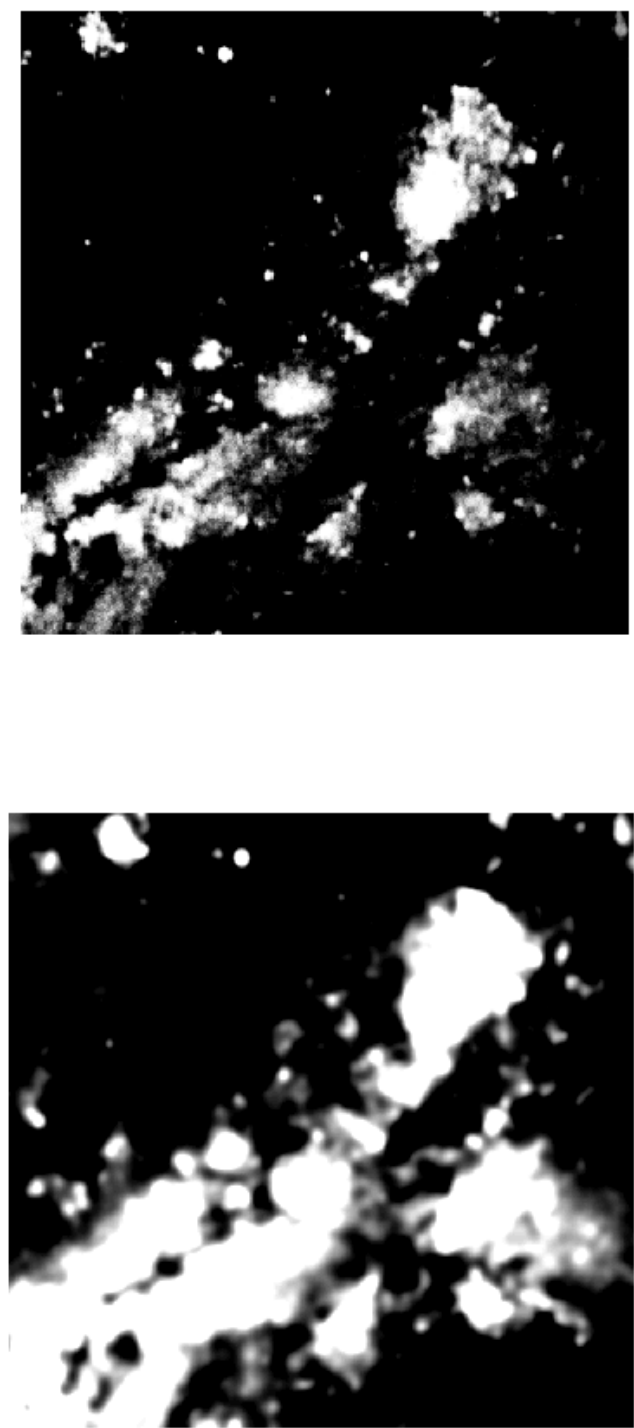

(d)

Fig. 1. An outer part of the Sc(s) I galaxy NGC 5457 covering an area of $4 \times 4 \mathrm{kpc}^{2}$ : a) digitised and inverted version of part of an enlargement of Plate 12 in AG (frame a); b) contrast enhancement of original; c) and d) Gaussian blurring followed by contrast enhancement; blurring over 2 pixels is applied 2 times and 4 times respectively

stellar formation and evolution mechanisms (star formation rates of different processes, rates of evolution of different galactic components, rates of massloss of different stellar species). The experiments described in the companion paper are indicative that such a scheme does indeed hold: The observed physical composition, and to a lesser extent the geometry, are found to be sensitive to the evolutionary parameters; moreover, over a reasonable parame- ter range the relevant observable magnitudes are found to be monotonically related to the evolutionary parameters.

As a final comment on the geometry we observe that the thickness of the disc of a typical galaxy is essentially of the same order as the size of the square region we are modelling. A fully satisfactory model should therefore be 3-dimensional. The present exploratory analysis carried out on a $2 D \mathrm{CA}$ must be regarded as provisional only. As transpires from the $3 D \mathrm{CA}$ experiments initiated by 
Comins (1983; Statler et al. 1983), the inclusion of the third dimension may indeed alter the quantitative conclusions drawn from $2 D$ simulations.

\section{Dynamics of the CA simulation}

(a)
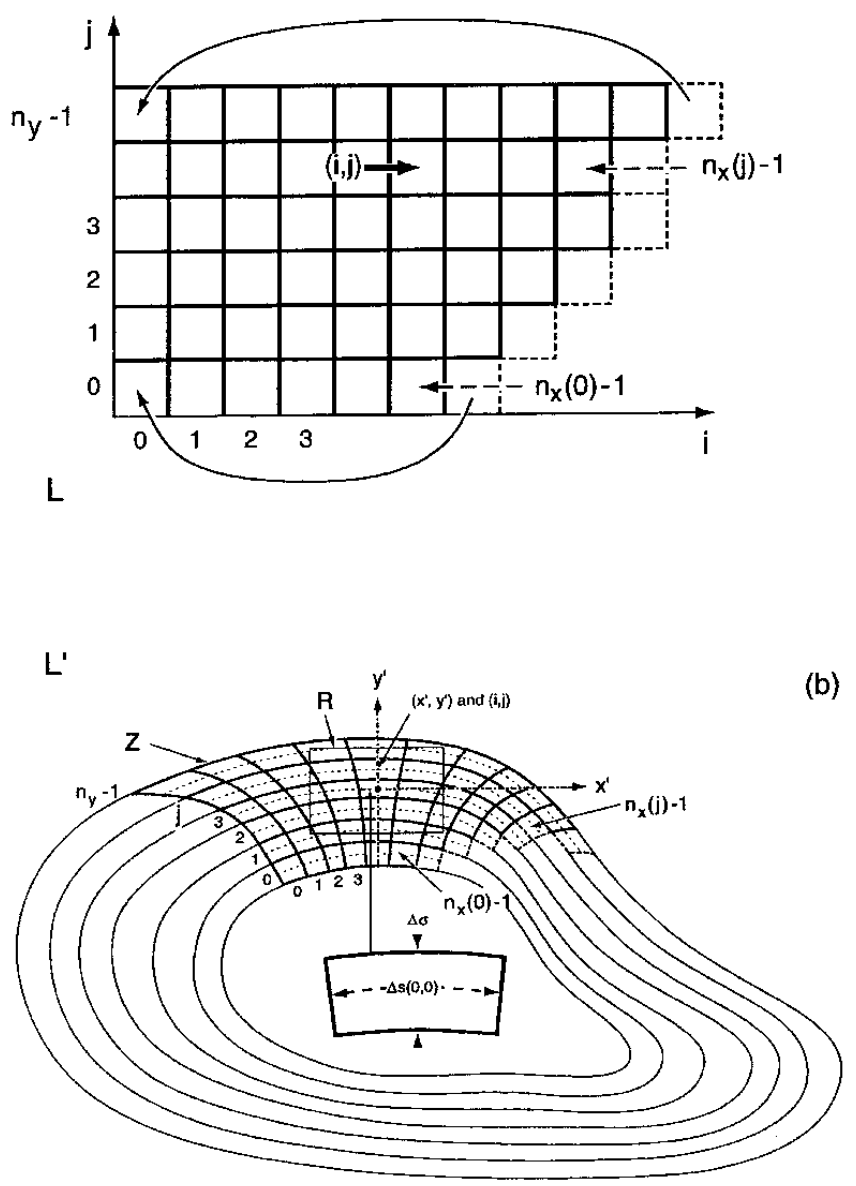

Fig. 2. a) Auxiliary square lattice $L ; i, j$ integer Cartesian coordinates; last dotted cell (right) to be identified with first cell (left) of each row $j=0,1,2, \ldots, n_{y}$. b) Lattice $L^{\prime}$ adapted to a (fictitious) galactic dynamics superposed on the space region (dotted square) to be modelled; $\mathbf{v}$, velocity field of Cartesian components $v_{x^{\prime}}\left(x^{\prime}, y^{\prime}\right), v_{y^{\prime}}\left(x^{\prime}, y^{\prime}\right) ; x^{\prime}, y^{\prime}$, Cartesian coordinates in frame $F$

The galactic dynamics is incorporated into our model through the following geometric procedure. We start out with an auxiliary $2 D$ lattice, $L$, of square cells (Fig. 2a) which assigns a cylindrical topology (possibly in the form of a conical geometry) to the configuration to be investigated; this topology takes care of the existence of local periods of the stationary galactic motion (cf. below). The geometrical structure of the true CA lattice, $L^{\prime}$, which represents the actual galactic region (Fig. 2b), is then encoded in a smooth deformation $D$ of the auxiliary lattice
$L, D: L \quad \rightarrow \quad L^{\prime}$. The mapping $D$ incorporates the dynamical effects of gravity-induced motions of the galactic configuration to be simulated.

The evolution calculations of the galactic components are performed on the auxiliary lattice $L$ and then visualised on the deformed lattice $L^{\prime}$. In order to avoid complications in the construction of the transformation $D$, we introduce two assumptions.

A1. The galactic velocity field $\mathbf{v}$ induced by the dynamics is such that there exists a relative reference frame $F$ in which the velocity is stationary.

This dynamic assumption requires a few comments.

If the velocity is a pure rotation of the galactic substratum, of rotational frequency $\omega=\omega(r)$ ( $r$, distance from galactic centre), then any uniformly rotating frame of centre coinciding with the galactic centre and of constant rotational frequency $\Omega$ (in particular $\Omega=0$ ), is a frame $F$. Assumption A1 thus covers the alternative studied in previous galactic CA models. More generally, under those circumstances any rotating frame of centre coinciding with the galactic centre and of arbitrary distance-dependent rotational frequency $\Omega=\Omega(r)$, can likewise be regarded as a frame $F$ (polar coordinates in $F,\left(r^{\prime}, \theta^{\prime}\right)$, related to the galactic polar coordinates $(r, \theta)$ by $\left.r^{\prime}=r, \theta^{\prime}=\theta-\Omega(r) t\right)$. As a further alternative, the streamlines may be circular but with an angular velocity depending on the azimuthal angle $\theta$ (rotation of local frequency $\omega=w(r) q(\theta)$ ); then on each streamline, identified by its radius $r$, matter is carried around the galaxy with a characteristic period $P^{*}(r)$. We shall restrict our attention here to planar velocity fields stationary in relative frames $F$ which are characterised in an algebraically simple way (for instance, $F$ uniformly rotating with respect to an absolute reference frame).

We have no guarantee that assumption A1 is applicable to real galaxies. For instance, if the macroscopic velocity field is turbulent then no reference frame of type $F$ exists. However, even under the latter extreme conditions, we may separate the actual velocity field $\mathbf{v}(t ; \mathbf{r})$ into a smooth, mean stationary component, $\mathbf{V}(\mathbf{r})$ in an appropriate relative reference frame $F$, and a time-dependent correction, $\nu(t ; \mathbf{r})$,

$$
\mathbf{v}(t ; \mathbf{r})=\mathbf{V}(\mathbf{r})+\nu(t ; \mathbf{r}) .
$$

The stationary component is then dealt with by the transformation procedures we are discussing here. The correction term, $\nu(t ; \mathbf{r})$, requires an ad hoc treatment. We have incorporated a diffusive mixing mechanism in our program to take care to some extent of nonstationary contributions.

The $2 D$ formulation imposes a topological constraint on the allowed stationary pattern of streamlines confined to a bounded area (the whole galaxy): In the plane the streamlines are either closed curves (proper streamlines), or they degenerate into singular points (isolated stable elliptic fixed points, or unstable hyperbolic [homoclinic or heteroclinic] fixed points). On a proper streamline the velocity field never vanishes; it always points in the same 
direction. Over each proper streamline, identified by a continuous coordinate $\sigma$, matter is periodically transported, with (finite nonzero) period $P=P(\sigma)$ depending on the streamline only. On a fixed point, $P \rightarrow 0$; on the streamlines connecting hyperbolic points, the period diverges, $P \rightarrow \infty$. To discard the latter complications a further kinematic assumption is in order.

A2. In the relative frame $F$ the region of the galaxy we are modelling contains proper streamlines only, and no fixed point.

Under A2 the deformation procedure is not applicable to a galactic region including the rotation centre (stable fixed point) as considered in MA and GSS. It is however consistent with the $2 \mathrm{D}$ models in C. ${ }^{9}$

Under assumptions A1, A2 we can introduce a change of space variables adapted to the geometry of the streamlines: The label $\sigma$ of the streamlines is identified with one new coordinate; the line element along a streamline, $d s$, defines a second coordinate $s$. The change from the space variables (say the Cartesian coordinates $\left(x^{\prime}, y^{\prime}\right)$ ) in $F$ to the new coordinates $(s, \sigma)$

$$
s=s\left(x^{\prime}, y^{\prime}\right) \quad \sigma=\sigma\left(x^{\prime}, y^{\prime}\right)
$$

is then interpreted as a transformation of reference frame, with $(s, \sigma)$ viewed again as Cartesian coordinates. In the new frame the stationary velocity field has parallel streamlines; the variable $s$ is cyclic.

To summarise, our geometrical treatment makes use of 3 different reference frames: (a) the physical frame ('absolute frame') such as the frame of an external observer with respect to which the photograph Fig. 1 is given; (b) the frame $F$ in which the galactic velocity is stationary; and (c) the auxiliary Cartesian frame of coordinates $(s, \sigma)$ in which the galactic streamlines are parallel.

\section{The coordinate transformation (4.2) in discrete form}

Suppose that the actual stationary velocity field is known (either observationally, or by solving an auxiliary dynamical problem). We denote by $v_{x}^{\prime}\left(x^{\prime}, y^{\prime}\right), v_{y}^{\prime}\left(x^{\prime}, y^{\prime}\right)$ and

\footnotetext{
${ }^{9}$ The approach continues to hold under the following less stringent requirement (consistent with MA and GSS) provided only that some precautions are taken: A2'. In the relative frame $F$ the region of the galaxy contains only proper streamlines and at most one fixed point. A stationary $2 D$ smooth vector field confined to a topological 2-disc of boundary defined by a proper streamline, and hence of degree 1 over the boundary of the disc, has $2 k+1$ fixed points, $k=0,1, \ldots$, among which at least $k$ are unstable; the minimum number of fixed points is thus 1 , and this fixed point must be elliptic (e.g. case of a differentially rotating galaxy whose centre is the stable fixed point). In the deformation procedure the elliptic point is modelled by a streamline reducing to a single cell, $\left(n_{x}=1\right.$, cf. Eq. (4.5)); the period $P$ of this formal streamline is then equal to the CA timestep $\Delta t ; P$ tends to zero in the limit $\Delta t \rightarrow 0$.
}

$v^{\prime}\left(x^{\prime}, y^{\prime}\right)(\equiv w(s, \sigma))$ the Cartesian components and the modulus of the velocity respectively, at any position of Cartesian coordinates $\left(x^{\prime}, y^{\prime}\right)$ in the stationary frame $F$. As origin of the frame we choose the centre $S$ of the region to be analysed; the axis $S x^{\prime}$ is parallel to the stationary velocity field at $S$ (positive $x^{\prime}$ direction = positive velocity direction); the axis $S y^{\prime}$ is directed outwards. With frame $F$ we attach a CA network (lattice $L^{\prime}$ ) of curved cells as illustrated in Fig. 2b. We identify a cell by the curvilinear coordinates $(s, \sigma)$ of its centre in $F$, or by a pair of integer Cartesian coordinates $(i, j)$ in the auxiliary square lattice $L$. The coordinates $\left(x^{\prime}(i, j), y^{\prime}(i, j)\right)$ in $F$ refer to the centre of the cell $(i, j)$. In the auxiliary lattice $L$ the image of the latter cell is identified by the integer Cartesian coordinates $(i, j)$ (Fig. 2a).

Figure $2 \mathrm{~b}$ shows on the one hand an aggregate of nested closed curves (drawn as continuous lines) representing streamlines in $F$ and defining layers of lattice cells in lattice $L^{\prime}$; these layers are the counterparts of the rings of cells in MA and GSS. The centres of the collection of cells are carried by a second aggregate of streamlines (drawn as dotted curves) issuing from a collection of points on the $y^{\prime}$ axis, which are cell centres, of coordinates $(0,0)$, $(0, \Delta \sigma(0)),(0,-\Delta \sigma(0)),(0, \Delta \sigma(0)+\Delta \sigma(1)),(0,-\Delta \sigma(0)-$ $\Delta \sigma(-1)), \ldots,(0, \Delta \sigma(0)+\Delta \sigma(1)+\ldots+\Delta \sigma(k)),(0,-\Delta \sigma(0)-$ $\Delta \sigma(-1)-\ldots-\Delta \sigma(-k)), \ldots$; the distance between successive centres, $\Delta \sigma(0), \Delta \sigma(1), \Delta \sigma(-1), \ldots, \Delta \sigma(k), \Delta \sigma(-k)$, $\ldots$, is chosen to secure conservation of mass (cf. below). The average 'height' $\Delta \sigma$ is a measure of the spatial resolution in the radial direction. The $y^{\prime}$ axis thus defines the zeroth column of cells.

The origin $S$ coincides with the centre of the cell identified by the integer coordinates $(i, j)=(0,0)$, and by the curvilinear coordinates $s=0, \sigma=0$. The line segment of origin $(0,0)$ and length $\Delta s(0,0)=v^{\prime}(0,0) \Delta t^{*}$ along the streamline in the positive velocity direction defines the cell centre of Cartesian coordinates $\left(x^{\prime}(1,0), y^{\prime}(1,0)\right)$ (right neighbour cell of cell $(0,0)$ ); the multiplier of the velocity, $\Delta t^{*}$, is a small enough reference dynamic timestep, which will be related to the true $C A$ timestep $\Delta t$. By iteration of this procedure we generate the centre of cell $(i+1,0)$, of Cartesian coordinates $\left(x^{\prime}(i+1,0), y^{\prime}(i+1,0)\right)$, from cell $(i, 0)$ of centre $\left(x^{\prime}(i, 0), y^{\prime}(i, 0)\right)$, by adding along the streamline a length $\Delta s(i, 0)=v^{\prime}\left(x^{\prime}(i, 0), y^{\prime}(i, 0)\right) \Delta t^{*}$. The construction is similarly continued in the negative velocity direction. We obtain in this way the zeroth row of cells. By an analogous construction applied to the streamline issuing from the $j^{\text {th }}$ point on the $S y^{\prime}$ axis, (centre of cell $(0, j)$, of Cartesian coordinates $\left.\left(0, y^{\prime}(0, j)\right)\right)$, we generate the $j^{\text {th }}$ row of cells. The $i^{\text {th }}$ column of cells is likewise the collection of cells $(i, 0),(i, 1),(i,-1)$, $(i, 2),(i,-2), \ldots$, of Cartesian coordinates $\left(x^{\prime}(i, 0), y^{\prime}(i, 0)\right)$, $\left(x^{\prime}(i, 1), y^{\prime}(i, 1)\right), \quad\left(x^{\prime}(i,-1), y^{\prime}(i,-1)\right), \quad\left(x^{\prime}(i, 2), y^{\prime}(i, 2)\right)$, $\left(x^{\prime}(i,-2), y^{\prime}(i,-2)\right), \ldots$ 
The lines issuing from $(0,-\Delta \sigma(0) / 2),(0, \Delta \sigma(0) / 2),(0$, $-3 \times \Delta \sigma(0) / 2),(0,3 \Delta \sigma(0) / 2), \ldots$, are again streamlines specifying the 'horizontal' boundaries of the rows of cells of the network $L^{\prime}$. The line midway between the centre points of columns $i-1$ and $i$ represents the common 'vertical' boundary between the cells of columns $i-1$ and $i$.

This construction provides a cellular network $L^{\prime}$ adapted to the stationary velocity field $\mathbf{v}(\mathbf{r})$ in frame $F$. By the same token it defines the correspondence between the lattice $L^{\prime}$ and the auxiliary lattice $L$ made up of identical unit square cells (cellsize $\Delta x=\Delta y=1$ ).

To avoid negative integer coordinates, it is convenient to choose as the origin $O$ of the auxiliary lattice $L$ the lower left corner cell; a cell of $L$ is then specified by $(i, j)$ with $i=0,1,2, \ldots, n_{x}(j)-1$ and $j=0,1,2, \ldots, n_{y}-1$; the number of rows (number of cells in the $y$-direction), $n_{y}$, is a parameter of the simulation $\left(\approx 10^{3}\right.$ in our experiments); the number of cells per row $j, n_{x}(j)$, depends on the streamline $j$ (cf. below). Henceforth a point $(i, j)$ in the auxiliary system $L$ will be understood as being defined in this latter system of nonnegative integer coordinates; the corresponding cell in the physical lattice $L^{\prime}$ (associated with the relative frame $F$ ) will be referred to by the same pair $(i, j)$.

The point of Cartesian coordinates $(i, j)$ in $L$ is mapped onto the point of Cartesian coordinates $\left(x^{\prime}, y^{\prime}\right)$ $=\left(x^{\prime}(i, j), y^{\prime}(i, j)\right)$ in $L^{\prime}$, of the cell of column $i$ and row $j$ in $L^{\prime}$ through the transformation $D$

$$
D: \quad(i, j) \mapsto\left(x^{\prime}, y^{\prime}\right)=\left(x^{\prime}(i, j), y^{\prime}(i, j)\right) ;
$$

explicitly, the Cartesian coordinates of the centre points of the cells of each row $j=0,1, \ldots, n_{y}-1$, in $L^{\prime}$ are given by the recurrence relations

$$
\begin{gathered}
x^{\prime}(i+1, j)=x^{\prime}(i, j)+v_{x}^{\prime}\left(x^{\prime}(i, j), y^{\prime}(i, j)\right) \Delta t^{*}, \\
y^{\prime}(i+1, j)=y^{\prime}(i, j)+v_{y}^{\prime}\left(x^{\prime}(i, j), y^{\prime}(i, j)\right) \Delta t^{*},
\end{gathered}
$$

$\left(i=0,1, \ldots, n_{x}(j)-1\right) \cdot{ }^{10}$ In the stationary hydrodynamic motion, the matter content at a position of Cartesian coordinates $\left(x^{\prime}, y^{\prime}\right)$ in the relative frame $F$ is shifted to the new position

$$
\left(x_{N^{\prime}}, y_{N^{\prime}}\right)=\left(x^{\prime}+v_{x}^{\prime}\left(x^{\prime}, y^{\prime}\right) \Delta t^{*}, y^{\prime}+v_{y}^{\prime}\left(x^{\prime}, y^{\prime}\right) \Delta t^{*}\right)
$$

in one dynamic timestep $\Delta t^{*}$, i.e. matter is just translated to the neighbour cell in the positive velocity direction. Denote by $N\left(Y ; t ; x^{\prime}, y^{\prime}\right)$ and $n\left(Y ; t ; x^{\prime}, y^{\prime}\right)$ the total amount of matter and the corresponding density respectively of species $Y$ at the continuous time $t$ in cell $\left(x^{\prime}, y^{\prime}\right)$ of $L^{\prime}$;

\footnotetext{
${ }^{10}$ If a stable fixed point is to be included (assumption A2'), we associate with it a single cell in $L^{\prime}$, of 'height' $\Delta \sigma(0,0)$ as above, and 'width' $\Delta s(0,0)=v^{\prime} \Delta t^{*}$; to avoid a flattened cell we identify $v^{\prime}$ with the average cell velocity of the closest proper streamline; this cell is then taken as the origin $(0,0)$ of the coordinate system, with $n_{x}(0)=1$. In the auxiliary frame $L$ the 'lowest' row then contains a single square cell.
}

denote further by $\Delta V\left(x^{\prime}, y^{\prime}\right)$ the (time-independent) 'volume' of this cell. The discrete CA time, denoted by $\tau$ (= $0,1,2, \ldots)$, is related to the continuous time by $t=\tau \Delta t$, $\Delta t$ being the automaton timestep; the relation between the kinematic timestep $\Delta t^{*}$ and the automaton timestep $\Delta t$ will be discussed below. In the physical lattice $L^{\prime}$ the constraint of conservation of species $Y$ in the mechanical motion, over one kinematic timestep $\Delta t^{*}$, becomes

$$
\begin{gathered}
N\left(Y ; t ; x^{\prime}, y^{\prime}\right) \equiv n\left(Y ; t ; x^{\prime}, y^{\prime}\right) \Delta V\left(x^{\prime}, y^{\prime}\right)= \\
n\left(Y ; t+\Delta t^{*} ; x_{N}^{\prime}, y_{N}^{\prime}\right) \Delta V\left(x_{N}^{\prime}, y_{N}^{\prime}\right) \equiv \\
N\left(Y ; t+\Delta t^{*} ; x_{N}^{\prime}, y_{N}^{\prime}\right) .
\end{gathered}
$$

In the auxiliary lattice $L$, the amount of matter in cell $(i, j)$ is just shifted to cell $(i+1, j)$ in one kinematic timestep $\Delta t^{*}$, so that the counterpart of (5.2) takes the simple form

$$
N(Y ; t ; i, j)=N\left(Y ; t+\Delta t^{*} ; i+1, j\right) .
$$

In this lattice the velocity $\left(=\Delta x / \Delta t^{*}\right)$ is a constant equal to 1 if time is measured in units of the kinematic step $\Delta t^{*}$.

The comparison of the treatment of a galactic rotation by the method initiated in MA with the deformed lattice technique as proposed here, illustrates clearly the advantage of the latter procedure. Under pure rotation the streamlines in the physical space are circular (the radius $r$ playing the role of the coordinate $\sigma$ ); the lattice $L^{\prime}$ is made up of concentric circular rings (labelled by $j$ ) subdivided into cells by roughly radially directed line segments; these segments make a progressively smaller angle with the rings as we go outwards, since the 'horizontal size' of the cells, $\Delta s=r \Delta \theta(r)(\Delta \theta(r)$, 'azimuthal size'), progressively increases with increasing distance $r$ from the galactic centre; cells of a ring centred on the same streamline have all same size. Since in the corresponding auxiliary lattice $L$ matter is shifted by one cell in each row $j$ per timestep $\Delta t^{*}$, to secure the correct period $P(j)$ on each proper streamline (row of cells) $j$, we have to select a number of cells per row $j, n_{x}(j)$, proportional to $P(j)$ (cf. Eq. (5.3) below).

In the procedures adopted by MA and GSS the circular rings defined by the streamlines are subdivided into cells which all have (approximately) the same width and area. To incorporate differential rotation, different rings are rotated by different amounts. If the lattice structure were preserved in the evolution, as a strict CA framework would require, then in one timestep the $j^{\text {th }}$ ring should be shifted by a fixed integer number of cells, $h_{j}=0,1,2, \ldots$, depending on the label $j$; two successive rings $j, j+1$ then would either corotate, $\left(h_{j+1}-h_{j}=0\right)$, or else suffer a relative shift of 1 , or $2, \ldots$ cells per timestep. In contrast, within the lattice deformation approach we simulate a much slower relative shift of ring $j+1$ with respect to ring $j$, namely of $1,2, \ldots$ cells per period $P(j)$. Hence a 
first advantage of the deformation procedure is that it can represent more accurately the differential rotation. ${ }^{11}$

In the second place, the deformation method is conceptually closer to the general spirit of CA models: Traditionally CA evolution rules are local rules, predicated on interactions between neighbour cells only. Unless special precautions are taken, the requirement of locality may be violated in the formal shift procedure, where two neighbour cells in adjacent rings may become widely separated in a single timestep (cf. the experiments in Lejeune \& Perdang 1991 which make use of the shifting method applied to a local part of the galaxy).

Thirdly, it is not clear how to extend the method of MA to arbitrary stationary motions. ${ }^{12}$

A closed streamline $j$ in the galaxy is simulated by the complete ring $j$ of cells of the physical lattice $L^{\prime}, n_{x}(j)$ being then the total number of cells in this ring. In contrast to the method of MA which models the whole galaxy, our purpose is to devise a procedure describing the behaviour of a small part of the galaxy only (rectangular zone $R$ in Fig. 2b), thereby avoiding an explicit treatment of the rest of the galaxy. Accordingly, we do not attempt to describe the whole ring but only a small section of it. To this end we embed $R$ in a larger region $Z$ (covered by the finite network $L^{\prime}$ ); $Z$ remains small as compared to the whole galaxy. The number of cells $n_{x}(j)$ thus represents the number of cells of ring $j$ within the region $Z$ in which we carry out our computations. The behaviour of the remainder of the galaxy is not explicitly treated; its effect is dealt with through boundary conditions at the border of zone $Z$.

In the case of a closed streamline $j$ in the relative frame $F$ (lattice $L^{\prime}$ ), the kinematic timestep $\Delta t^{*}$ and the period $P(j)$ associated with this streamline, determine the total number of cells, $n_{x}(j)$, in the full ring $j$ defined by this streamline, namely $n_{x}(j) \approx P(j) / \Delta t^{*}$. In a rigorous

\footnotetext{
${ }^{11}$ In practice, the accuracy in the ring shifting method is improved by actually shifting successive rings by fractional amounts of the cell size; however, such a procedure does not conform to a standard CA methodology.

${ }^{12}$ In the deformation method the stationarity assumption A1 of the velocity field in the $2 D$ galactic model, and the kinematic assumption A2 (or A2') appear as essential ingredients. Only under these assumptions do we secure a simple geometry of the streamlines, and hence the existence of a geometrically simple auxiliary lattice $L$. In the presence of several fixed points (violation of A2') the elementary structure of an auxiliary lattice $L$ is not only destroyed: it also raises the problem of handling streamlines connecting unstable fixed points. We should finally stress the special constraint imposed by the dimensionality 2 of our simulation. In a $3 D$ space stationarity of a velocity field confined to a bounded region does not automatically imply periodicity; stationary streamlines in $3 D$ confined to a bounded region may not close, in which case they either correspond to multi-periodic, or else to chaotic transport of matter. It is not clear how the latter alternative could be simulated kinematically in a CA environment.
}

treatment the full ring should be dealt with; in the latter, cell $i=0$ is identical with cell $i=n_{x}(j)$, a property which can be interpreted as a boundary condition. In our local formulation $n_{x}(j)$ represents a fraction $f \approx 1 / K(K$, an integer independent of $j$ if the region $Z$ is properly chosen) of the total number of cells of the closed streamline $j$. The number of cells in the section of the streamline lying in $Z$ is thus given by

$$
n_{x}(j) \approx \frac{P(j)}{K \Delta t^{*}} .
$$

This procedure is consistent with the true period for the transport of matter on the streamline $j$. In the present case an explicit boundary condition is needed; to this end, just as for the full ring, we identify cells $i=0$ and $n_{x}(j)$ on each streamline $j$ (cf. the auxiliary lattice Fig. 2a where the dotted cell on the right is identified with the first cell on the left). ${ }^{13}$

The boundary conditions we are then led to adopt are

$$
\text { state of cell }\left(n_{x}(j), j\right) \equiv \text { state of cell }(0, j) \text {, }
$$

state of cell $(-1, j) \equiv$ state of cell $\left(n_{x}(j)-1, j\right)$.

The latter condition is needed when dealing with the neighbourhood of cell $(0, j) \cdot{ }^{14}$

At the bottom $(j=0)$ and top rows $\left(j=n_{y}-1\right)$ we impose artificial reflection conditions. The 'lower' neighbour of a cell in the bottom row, $(i, 0)$, is identified with its 'upper' neighbour; and the 'upper' neighbour of a cell of the top row, $\left(i, n_{x}\right)$ is identified with the 'lower' neighbour of this cell

$$
\begin{gathered}
\text { state of cell }(i,-1) \equiv \text { state of cell }(i,+1), \\
\text { state of cell }\left(i, n_{y}\right) \equiv \text { state of cell }\left(i, n_{y}-2\right) \text {. }
\end{gathered}
$$

For an endcell $\left(n_{x}(j)-1, j\right)$ of a row, the 'lower' and 'upper' neighbours are read off from Fig. 2a. The latter conditions are required for the specification of the neighbourhood of a boundary cell. ${ }^{15}$

\footnotetext{
${ }^{13}$ Geometrically this imposes a fictitious $K$-fold symmetry to the global structure of the galaxy; but since the modelling procedure only deals with $1 / K$ of the ring, this symmetry never becomes observable in the simulation. With $K$ integer, the true periodicity $P(j)$ remains an exact period of the motion along the streamline.

${ }^{14}$ The approach we have adopted is reminiscent of a method applied in statistical mechanics: instead of computing for instance the behaviour of a gas in a large vessel, the gas is formally confined to a small cubic test region, and simple periodicity conditions are imposed on the surface of the cube; the idea is that the precise form of the boundary conditions does not influence significantly the behaviour inside the test cube.

${ }^{15}$ We should keep in mind that since neighbour rows have generally different numbers of cells $n_{x}(j)$, after a time interval $P(j) / K$ row $j$ is shifted with respect to its neighbour rows. Matter initially in say column 0 thus distributes over several
} 


\section{CA simulation of star formation and stellar evolution}

As in our previous simulations (Perdang 1990; Lejeune \& Perdang 1991; Lejeune 1993), the following galactic species are dealt with in the evolutionary computations: interstellar gas and dust, $D$, (in the form of molecular clouds); massive protostars, $P$; massive main sequence stars, $M$, massive evolved stars (referred to as 'red giants') $R$; inert remnants (white dwarfs, neutron stars, black holes), $I$; and low mass stars, $L$. A given cell $(i, j)$ in lattice $L^{\prime}$ or in the auxiliary lattice $L$, is either empty, $E$, or populated by at most one of the 6 species listed. The field function $F(\tau ; i, j)$ encoding the state of cell $(i, j)$ at discrete time $\tau$, is defined over $\mathbb{Z}_{7} ;$ (7-state automaton; $F=0$ : empty state $E$, black in our colour plates; $F=1$ : gas state $D$, cyan; $F=2: P$, white; $F=3: M$, yellow; $F=4: R$, red; $F=5: I$ blue; and $F=6: L$ magenta).

There is manifestly considerable freedom in the definition of 'massive' and 'low mass' stellar objects. We have here in mind representative orders of magnitude for the $P$, $M$ and $R$ objects of $15-20 M_{\odot} ; L$ objects are assigned masses of about $1 M_{\odot}$ or lower. The choice of a mass range $>15 M_{\odot}$ for massive stars is dictated by the requirement that the surface brightness of the galaxy be dominated by the luminosity of these objects (in their early evolutionary phase $P$; luminosity $>10^{5} L_{\odot}$ for $\left.>15 M_{\odot}\right)$. On the other hand, with the assignment of a mass of $1 M_{\odot}$ to species $L$, low mass stars do not significantly evolve over the timespan of an experimental run (not substantially exceeding $10^{8} \mathrm{y}$ ); for the purposes of evolution both states $L$ and $I$ then play the parts of sinks for the galactic material. Over the same timespan of one run a massive star goes through a full evolutionary cycle; at the end of the cycle most of the star's matter is returned into the active gas form $D$; a new cycle then starts all over again.

If we adopt a surface density of matter of the order of the solar neighbourhood density, $\left(\approx 75 M_{\odot} / \mathrm{pc}^{2}\right.$; Binney $\&$ Tremaine 1987), then a cell of our $10^{3} \times 10^{3}$ automaton (simulating a total area of $16 \mathrm{kpc}^{2}$ ), if not empty, carries an average mass of roughly $10^{3} M_{\odot}$; the cell has a size of the order of the size of a cluster or an association. If in a $P, M$ or $R$ state, then the cell contains approximately 50 actual protostars, main sequence stars or red giants; if in an $L$ or $I$ state, it contains $10^{3}$ low mass or inert stars.

columns after a time $>P / K$, where $P$ is the period associated with any streamline. Under the amended assumption A2', in the presence of a stable fixed point the right and left neighbours of the corresponding cell $(0,0)$ are to be identified with the cell $(0,0)$ itself, in conformity with Eq. (5.4); the shift of matter to the neighbour cell in one timestep thus leaves this cell unchanged. The region in physical space containing the fixed point surrounds the latter; in the simulation on lattice $L^{\prime}$ cell $(0,0)$ is thus surrounded by a ring of 8 cells, with the first and last cells of the ring being physical neighbours; the periodicity condition (5.4) is then exact.
With our evolution processes being handled on the level of cellstates rather than on the level of actual galactic objects, the physical parameters referring to stars (transition probabilities per timestep) must be rescaled to individual cells. ${ }^{16}$

The stellar evolutionary processes accounted for in our model are transformations among the 6 galactic species, simulated by transitions among the 6 non-empty cellstates of our CA model. These transformations are conveniently visualised as chemical reactions symbolised by stoichiometric schemes of the form

$$
\begin{gathered}
X+n C+m K+\ldots \rightarrow \\
Y+n C+m K+\ldots, \quad P[X, n C, m K, \ldots ; Y, n C, m K, \ldots] .
\end{gathered}
$$

This notation means that if at discrete time $\tau$ cell $(i, j)$ is in state $X$, and $n$ cells $\left(i^{\prime}, j^{\prime}\right),\left(i^{\prime \prime}, j^{\prime \prime}\right), \ldots$, of the neighbourhood $\mathbb{N}(i, j)$ of cell $(i, j)$ are in state $C, m$ cells of the neighbourhood are in state $K$, etc, then at discrete time $\tau+1$ state $X$ is replaced by state $Y$ with probability $P[X, n C, m K, \ldots ; Y, n C, m K, \ldots]$. The neighbourhood $\mathbb{N}(i, j)$ we adopt is the Moore neighbourhood, consisting of the cell $(i, j)$ itself and its 8 neighbours $(i, j \pm 1),(i \pm 1, j)$ and $(i \pm 1, j \pm 1)$. Species $C, K, \ldots$ act as catalysts. In this section we regard times are being expressed in units of an evolutionary timestep, $\Delta t^{* *}$, adapted to the evolutionary timescale of our massive objects, $\Delta t^{* *} \approx 510^{5} \mathrm{y}$.

The initial state of the galaxy is a diffuse and spatially non-uniform aggregate of gas: any cellstate $F(0 ; i, j)$ is either 0 ( $E$ state), or 1 ( $D$ state) (cf. Paper II).

\subsection{Star formation}

The two protostar formation processes already implemented in the model of MA as well as in all subsequent CA models are naturally included in our formulation:

$(\alpha)$ Spontaneous gravitational collapse of interstellar matter (molecular clouds) into protostars, as a result of a Jeans instability.

We regard the latter as being operative at a given site in the galaxy provided that a large enough neighbourhood contains interstellar matter; essentially the latter proviso guarantees that a critical wavelength may actually be realised in the neighbourhood of the site. Spontaneous star formation produces both low mass stars and massive stars. The corresponding stoichiometric schemes are

$$
\begin{array}{lll}
D+d D & \rightarrow P+d D ; & P[D d D ; P d D] \\
D+e D & \rightarrow L+e D ; & P[D e D ; L e D]
\end{array}
$$

we have set $d \geq 5$ and $e \geq 2$. The probabilities of these processes are chosen small enough, $510^{-5}$ ( $P$-formation)

${ }^{16}$ With $10^{3} P$ cells in a $10^{3} \times 10^{3}$ automaton (about $0.1 \%$ of the matter in $P$ form) the average luminosity $/ \mathrm{pc}^{2}$ for a region of $4 \times 4 \mathrm{kpc}^{2}$ is $\approx 300 L_{\odot} / \mathrm{pc}^{2}$ (order of central surface brightness




and $1.710^{-4}$ ( $L$-formation) per timestep $\Delta t^{* *}$; the precise value of the $P$-formation rate by process $(6.2)$ is found to be immaterial for the actual evolution of the galactic matter; this process is indeed obliterated by star-induced star formation $(\beta)$, as soon as a few protostars are present in the medium; the role of a small transition probability of (6.2) is to delay the efficient onset of star formation through the explosive catalytic process $(\beta)$ discussed below. Test experiments, with an initial state inseminated by just a few protostars $\left(\approx 10\right.$ in a lattice of $10^{6}$ cells $)$ and with process (6.2) entirely suppressed, demonstrate that the subsequent evolution of the galactic species is virtually identical with the original evolution.

( $\beta$ ) Catalytic protostar formation. The global stoichiometric scheme symbolising this process is

$$
\begin{gathered}
D+p P+m M+r R \rightarrow \\
P+p P+m M+r R ; P[D p P m M r R ; \operatorname{Pp} P m M r R]
\end{gathered}
$$

which is our counterpart of the more schematic process (1.2) of MA. Since catalytic star enhanced star formation constitutes the main ingredient of SPSF, advocated in all CA simulations of galactic structure (cf. GSS), it is superfluous to rehash the arguments in favour of this mechanism. In our numerical implementation we require that $p+r \geq 1$, or $m \geq 4$, expressing that luminous young objects or luminous evolved stars (which end as supernovae) have a far more efficient triggering action than massive main sequence stars (a Moore neighbourhood with at least $4 M$ cells having a virtually vanishing probability to materialise).

The numerical values we have adopted for the transition probability of process (6.4) lie in the range $0.03-0.3$ per timestep $\Delta t^{* *}$. In GSS this probability is the main model parameter responsible for the onset of the percolation phase transition.

\subsection{Stellar evolution}

Besides the usual evolutionary steps of isolated stars

$$
\begin{array}{cc}
P \rightarrow M ; & P[P ; M], \\
M \rightarrow R ; & P[M ; R], \\
R \rightarrow I ; & P[R ; I], \\
L \rightarrow I ; & P[L ; I],
\end{array}
$$

whose transition probabilities are provided by standard stellar evolution, we add artificial steps of disintegration for each 'active' stellar species $A$ into gas

$$
A \rightarrow D ; \quad A=P, M, R \text { or } L ; \quad P[A ; D]
$$

the latter simulate mass loss accompanying the normal evolution of all massive stars, as well as the cataclysmic late evolutionary stages.
The orders of magnitude of the transition probabilities per timestep we adopt are $P[P ; M] \approx 0.002 ; P[M ; R] \approx$ $0.02 ; P[R ; I] \approx 510^{-4} ; P[L ; I] \approx 410^{-6}$; with the latter 'disintegration' probability of the $L$ objects this mechanism is inoperative over the timescale of our experiments. The estimate of the transition probabilities is illustrated in the case of $P[R ; I]:$ A genuine massive red giant transforms about $1 / 15^{\text {th }}$ to $1 / 20^{\text {th }}$ of its matter into inert objects, the remainder being recycled as gas; if we take a timescale of $510^{7} \mathrm{y}$ for the massive red giant phase, since 15 to $20 R$ cells are needed to form one $I$ cell, the effective timescale for the formation of one $I$ cell becomes $\approx 10^{9} \mathrm{y}$; hence we have a probability per timestep of the order of $\Delta t^{* *} / 10^{9} \approx 510^{-4}$.

As tentative orders for the probabilities of mass loss we adopt $P[P ; D] \approx 0.09 ; P[M ; D] \approx 0.025 ; P[R ; D] \approx$ $0.1 ; P[L ; D]<0.0001$.

In addition to the standard stellar evolutionary steps (6.5-8) we include catalytic steps which are meant to reflect the property that in a close multiple stellar system the evolution of an individual component is affected by its companions (essentially through exchange of mass). We include the following schemes

$$
\begin{gathered}
P+p P+m M+r R \rightarrow \\
M+p P+m M+r R ; \quad P[P p P m M r R ; M p P m M r R] \\
M+r R \rightarrow R+r R ; \quad P[M r R ; R r R] .
\end{gathered}
$$

The first reaction mechanism, which we let operate if $p+r \geq 1$ or $m \geq 4$ (cf. above), captures the transformation of a protostar into a main sequence star under the effect of the extra radiation field of the companions; we have here tentatively set this probability equal to 0.05 . The second process (with $r \geq 1$ ) symbolises the evolution of a main sequence star in a close binary or in a higher order multiple stellar system. The transformation rate of the main sequence star into a red giant in the presence of one or several more evolved companions is speeded up, as a result of mass transfer from the evolved $(R)$ to the less evolved body $(M)$. In our test experiments we have typically chosen $P[M r R ; R r R] \approx 0.075$. Physically the evolution of a main sequence star in a close binary is not a genuine catalytic step, since both components, the main sequence star and the red giant undergo mutually induced evolutionary changes. By formally approximating this evolutionary stage by (6.11) we disregard the fact that while suffering mass loss, the $R$ cell suffers a change in its evolutionary rate as well; the reaction step (6.11) only duplicates the effect of mass accretion on the $M$ cell whose evolution thereby may be affected significantly.

It was noticed in the context of simplest ODE models (1) that the combined presence of the catalytic steps (6.4) and (6.11) in the network of evolutionary transformations can lead to oscillations in the global average of the population of the different species (Perdang 1974). The numerical 
experiments carried out with the present CA model not only confirm the possibility of a periodic time behaviour in the spatially extended system, for specific choices of the parameters; they also indicate that a transition to spatial inhomogeneity can take place, reminiscent of the spatial symmetry breaking in reaction-diffusion systems and induced by an instability in the reaction scheme (Prigogine \& Lefever 1968; Glansdorff \& Prigogine 1971). In chemical reactions one necessary condition for any symmetry breaking, temporal or spatial, is the presence of catalytic steps in the stoichiometric equations; a second quantitative condition is that the timescales in the different reaction steps inducing the instability be of same order. Under usual conditions prevailing in a galaxy, the latter requirement is probably not fulfilled, so that this dynamic regime seems to be of marginal astrophysical interest only.

\subsection{Other effects}

The model includes an inflow of gas from the galactic halo onto the disk during the early galactic evolution. This is simulated by a gas 'creation' scheme

$$
E \rightarrow D, \quad P[E ; D]
$$

The corresponding transition probability is set equal to $110^{-5}$, during the first 100 evolutionary timesteps (contraction phase of galaxy); it drops to zero at later times.

As an admittedly rough way of accounting for proper motions, as well as for non-stationary irregular hydrodynamics, we implement a spatial diffusion or mixing mechanism affecting all of the galactic species in the same way. As in our previous papers, this is done via the simple algorithm of Chopard \& Droz (1990). The random quasiisotropic mixing is applied in the auxiliary lattice $L$, so that in the physical frame $L^{\prime}$ isotropy of the irregular motions appears as broken, as it should do.

For the simplest evolution processes of isolated stars (6.5-6.8), stellar evolution theory supplies, in principle, reliable timescales for individual masses. The relevant transition probabilities for our model are averaged inverse timescales over the stellar mass distribution. Our artificial subdivision of the stellar mass spectrum into just two categories, low mass stars and massive stars, makes these probabilities strongly dependent on the specific mass at which the subdivision occurs; moreover these probabilities depend on the precise form of the mass spectrum and especially on cut-offs. This leaves considerable uncertainty even in these transition probabilities. The characteristic times for star formation, for the processes of mass loss, and for the catalytic steps $(6.10,11)$, can only be guessed at; the 'reaction rates' for these processes must be regarded as free parameters. The main goal of the present work is to show that physical and geometrical observational information (relative amounts of different galactic species, local fractal dimension of arms, etc, in real galaxies) may be used to constrain these parameters.

\section{Stationary velocity field of the CA model}

The velocity pattern we select (over a square area $R$ of about $16 \mathrm{kpc}^{2}$, of centre at a distance $r_{\mathrm{S}}=6 \mathrm{kpc}$ from the galactic centre) is required to possess characteristic properties generic for actual galactic fields. Therefore we choose a velocity field which is rotational on average, of the order of $200 \mathrm{~km} \mathrm{~s}^{-1}$, and which increases outwards by several $\mathrm{km} \mathrm{s}^{-1}$ per kpc. In an absolute reference frame our ansatz for the angle-averaged azimuthal velocity at a distance $r$ from the galactic centre $O$ is

$$
\begin{gathered}
v_{o}(r)=v_{\mathrm{a}}+\left(r-r_{\mathrm{S}}\right) v_{\mathrm{b}}^{\prime}, \\
v_{\mathrm{a}} \approx 200 \mathrm{kms}^{-1}, \quad v_{\mathrm{b}}^{\prime} \approx 3.5 \mathrm{kms}^{-1} / \mathrm{kpc} .
\end{gathered}
$$

We assume that the complete velocity field differs from the average field by correction factors less than $10 \%$ of the value of $v_{\mathrm{a}} \cdot{ }^{17}$

In conformity with assumptions $\mathrm{A} 1, \mathrm{~A} 2$ we postulate the existence of a relative reference frame $F$ in which the true velocity field is stationary and has no fixed points. We approximate this reference system by a frame in uniform rotation with respect to the absolute frame; the order of magnitude of the period $P_{\mathrm{F}}$ of frame $F$ with respect to the absolute frame will be chosen close enough to $P(S)$; within $F$ the angle-averaged velocity then becomes

$$
v_{o}^{*}(r)=v_{\mathrm{a}}^{*}+\left(r-r_{\mathrm{S}}\right) v_{\mathrm{b}}^{\prime}, \quad v_{\mathrm{a}}^{*} \ll v_{\mathrm{a}}, \text { in } F
$$

(without loss we require $v_{o}{ }^{*}(r)>0$ in the region to be modelled). The absence of fixed points is secured if we have

$$
v_{\mathrm{a}}^{*}>\left(r_{\mathrm{S}}-r_{\min }\right) v_{\mathrm{b}}^{\prime} \text { or } v_{\mathrm{a}}^{*}>7 \mathrm{~km} / \mathrm{s}
$$

for the orders of magnitude adopted.

We describe the full (non-averaged) stationary velocity field in zone $Z$ embedding $R$ in polar coordinates, by a Fourier-type ansatz of the form

$$
\begin{gathered}
V_{\theta}(r, \theta)=v_{o}{ }^{*}(r)+ \\
v_{1}(r) \sin \left(2 \pi \theta / \Theta(r)+\Phi_{1}(r)\right)+v_{2}(r) \sin \left(4 \pi \theta / \Theta(r)+\Phi_{2}(r)\right) \\
V_{\mathrm{R}}(r, \theta)=w_{1}(r) \sin \left(2 \pi \theta / \Theta(r)+\phi_{1}(r)\right)+ \\
w_{2}(r) \sin \left(4 \pi \theta / \Theta(r)+\phi_{2}(r)\right)
\end{gathered}
$$

${ }^{17}$ These orders of magnitude are consistent with the rotation velocity in our Galaxy $\left(v_{\mathrm{a}}=220 \mathrm{~km} \mathrm{~s}^{-1}\right.$ for solar neighbourhood, distance $\approx 8.5 \mathrm{kpc} ; A$-value of galactic rotation curve: $13.3 \pm 1.5 \mathrm{~km} \mathrm{~s}^{-1} / \mathrm{kpc}$, Hron \& Maitzen 1985 ; we have chosen here a somewhat smaller gradient). With the average rotational velocity it takes $210^{7}$ y for matter on the streamline passing through the centre $S$ to be shifted from one side to the other in our model. This corresponds to $1 / 10^{\text {th }}$ of the local period $P(S)$ (rotation period of solar neighbourhood: $2.410^{8} \mathrm{y}$ ). 
(origin of the coordinate system: centre of galaxy; $\theta=0$ : direction of centre $S$ of the modelled region). ${ }^{18}$ The amplitudes $\left(v_{1}(r), v_{2}(r), w_{1}(r), w_{2}(r)\right)$ we adopt in an actual numerical experiment are generated by the following random process. If $\nu$ denotes a random number of uniform distribution in $(-1,1)$, we assign to each of these amplitudes $u(r)$ an expression

$$
u(r)=\nu \times U \times\left(r / r_{\mathrm{S}}\right)^{\alpha}, U \sim 10 \mathrm{kms}^{-1} ;
$$

this order of magnitude, a fraction of $10 \mathrm{~km} \mathrm{~s}^{-1}$, is observationally reasonable. For the phases $\Psi(r)\left(\Phi_{1}(r), \Phi_{2}(r)\right.$, $\left.\phi_{1}(r), \phi_{2}(r)\right)$ we similarly set

$$
\Psi(r)=\nu \times \pi \times\left(r / r_{\mathrm{S}}\right)^{\beta} .
$$

The function $\Theta(r)$ is a typical angular size over which the field changes; to account for spatial inhomogeneities of a linear size of $1 \mathrm{kpc}$, we set

$$
\Theta(r)=T \times\left(r / r_{\mathrm{S}}\right)^{\gamma}, T \sim 1 .
$$

In representations (7.2a-c) the parameters $\alpha, \beta, \gamma$ are positive reals. A realisation of a 2 -dimensional velocity field (7.2a-c) in the relative frame $F$ is exhibited in Fig. $3\left(v_{\mathrm{a}}^{*}\right.$ $=12 ; v_{\mathrm{b}}^{\prime}=0.5 ; \alpha=3 ; \beta=1 ; \gamma=2$; amplitude factors $U=10, T=0.7)$. Besides the shearing effect, this field shows a change in curvature and it is slightly diverging towards the right.



Fig. 3. Random realisation of a velocity field defined by Eqs. $(7.2)$

Our purpose of including a schematic simulation of details finer than a mere differential rotation in the galactic kinematics is to examine whether the latter influence the quantitative aspects of actual galactic evolution in a significant way. Test runs corresponding to different stochastic realisations of the amplitudes and phases (7.2) indicate that the qualitative large scale structure of the spatial patterns generated by our CA model, reflects to some

\footnotetext{
${ }^{18}$ The angle-dependent corrections in Eq. (7.2) are meant to approximate the field over the local region $Z$; the approximation is not required to hold outside $Z$. Therefore the angular averaging of the specific expressions (7.2) need not vanish.
}

extent these kinematic details. In contrast, the quantitative parameters specifying the fine structure of these patterns (fractal dimension of isophotes, fractal dimension associated with distribution of evolved stars) are only marginally dependent on the details of the non-rotational velocity contributions. The rotational shear effect in the angle-averaged velocity field in $F$ (Eq. 7.1a) dominates the dynamical shaping of the geometrical fine structure of galactic arms, as was already clear from MA.

\section{Choice of the CA timestep}

With a lattice of about $10^{3}$ cells in the radial direction and $10^{3}$ cells in the azimuthal direction, the height and angular size of a cell are $\Delta r \approx 4 \mathrm{pc}$ and $\Delta \theta \approx 710^{-4}$; the width at distance $r_{\mathrm{S}} \approx 6 \mathrm{kpc}$ from the galactic centre is $\Delta x \approx 4 \mathrm{pc}$. In the absolute reference frame, the typical average rotation velocity is such that matter is shifted a distance of $4 \mathrm{pc}$ in a time interval of $210^{4} \mathrm{y}$. The latter value would then fix the order of magnitude of the $d y$ namic timestep $\Delta t^{*}$ of our model, if we were to identify system $F$ with the absolute reference system. However, the evolutionary timestep $\Delta t^{* *}$ adapted to the numerical simulation of the stellar evolutionary processes is about 25 times longer than the above value for $\Delta t^{*}$, so that such a step is not adapted to stellar evolution. On the other hand, we may identify the $C A$ timestep $\Delta t$ with the evolutionary timestep $\Delta t^{* *}$;

$$
\Delta t \equiv \Delta t^{* *}=510^{5} \mathrm{y} .
$$

With this choice it is possible to secure that matter be transferred on average from one cell to the neighbour cell in one CA timestep, i.e. it is possible to choose a dynamic timestep

$$
\Delta t^{*} \equiv \Delta t .
$$

Indeed, we achieve this goal by selecting as the relevant reference frame $F$ a uniformly rotating frame, of rotational speed such that the average velocity of level $S$ is equal to $\Delta x / \Delta t \approx 8 \mathrm{~km} / \mathrm{s}$ in this frame. We then have for the constant velocity parameter in Eq. (7.1.a) $v_{a}^{*} \equiv \Delta x / \Delta t$. This value remains perfectly consistent with assumption A2 (Eq. 7.1b).

\section{Final remarks}

The model discussed in this paper is essentially a continuation of the line of approach initiated in MA. It incorporates two new features not dealt with in the currently available galactic models. In the first place the model formulation enables us to incorporate any quasi-stationary overall galactic velocity field, through the introduction of a deformed lattice, while currently available CA models are based on a radial symmetry of the kinematics. In the second place, the model includes an already detailed though schematic picture of stellar evolution (essentially 
absent from current CA models) interpreting the individual galactic components as chemical species undergoing reactions. There is no technical difficulty for adding finer stellar evolutionary effects (subdivision of stars into different mass classes, ...). The elementary version of the present model contains only a modest number of free parameters which, as is shown in the companion paper, are recoverable, in principle, from observation.

An attractive feature of our formulation is that it follows the evolution of a local zone of the galaxy without explicitly treating the rest of the galaxy.

Being an extension and generalisation of previously proposed CA models, the present model can certainly simulate spiral structure (parts of local arms in the local version) for a proper range of the evolutionary parameters. In fact, our model incorporates a mechanical effect not dealt with in previous CA formulations which contributes to the formation of arms: Since our initial conditions correspond to a non-uniform matter distribution in space, any stationary shearing motion, simulated in our model by the combined effect of the deformation of the lattice and the progressive shift of neighbour streamlines necessarily produces a spiral arrangement of the non-uniformities in the background matter. This effect was already demonstrated by Heisenberg \& von Weizsäcker (1948) in a schematic $N$-body simulation. The occurrence of 'luminous' spirals, superposed over this background, requires that the high luminosity objects (mainly the $P$ components of our simulation) obey two conditions: i) they form in bunches, which is secured by SPSF (formation rate 6.4); and ii) they do live long enough but not too long, which is regulated by the various decay rates of the $P$ component (Eqs. 6.5, 6.9, $6.10)$.

As a final remark, we wish to insist that a CA model is a device 'simulating' the real physics rather than conforming to the details of the physical mechanisms. For instance the specific implementation of the reaction scheme (6.4) can be interpreted as the effect of a shock propagation, say from a supernova. In our model a single shock then propagates with a speed $\approx 10 \mathrm{~km} / \mathrm{s}$ (an order of magnitude observationally acceptable, cf. Comins 1981); this shock is in principle efficient to trigger the formation of up to 400 stars (a large open cluster), concentrated over the 8 cells (area of $100-200 \mathrm{pc}^{2}$ ) surrounding the shock centre; the reaction rate itself is a free parameter which can be adjusted to avoid observationally inconsistent outcomes. The model thus realistically mimics the effect of the shock mechanism, even though the spatial resolution available does not enable us to follow the propagation of the real shock.

If on the scale of individual cells a CA model result were to appear as inacceptable, we should keep in mind that a CA simulation cannot resolve the physics on the microscopic level of individual cells; comparisons with observations are physically meaningful only in the form of averages over a sufficient number of cells. It is for this reason that numerical results of a CA calculation should be presented in some smoothed form (cf. Paper II).

Acknowledgements. The authors are indebted to G. Barbaro for helpful comments and for relevant information on the parameters related to stellar evolution; they thank S. Tremaine for a helpful discussion, D. Lynden-Bell for his comments on M101, and especially H. Robe for his careful reading of an early version of the manuscript. They also thank R. Sword for redrawing Fig. 2. They wish to thank the referee, Dr N. Comins, for relevant comments which helped improve the presentation of this work. J.P. acknowledges a Royal Society-FNRS European Exchange Grant and financial support from the FNRS (Belgium).

\section{References}

Aarseth S.J., 1972, in: Gravitational N-Body Problem, IAU Colloquium 10. Lecar M. (ed.). Reidel, Dordrecht, p. 373

Arnett W.D., Bahcall J.N., Kirschner R.P., Woosley S.E., 1989, ARA\&A 27, 629

Arnold V.I., 1976, Méthodes mathématiques de la mécanique classique, Mir, Moscow

Balser D.S., Comins N.F., 1988, ApJ 328, 499

Bazell D., Désert F.X., 1988, ApJ 333, 353

Berkhuijsen E.M., 1974, A\&A 35, 429

Bertin G., Lin C.C., Lowe S., Thurstans R., 1989, ApJ 338, 78

Binney J., Tremaine S., 1987, Galactic Dynamics. Princeton University Press, Princeton

Blacher S., Perdang J., 1990, Vistas Astron. 33, 393

Brandstadter A., Swinney H.L., 1987, Phys. Rev. A35, 2207

Castor J., McCray R., Weaver R., 1975, ApJ (Lett.) 200, L107

Chopard B., Droz M., 1990, in: Cellular Automata and Modeling Complex Physical Systems, Springer Proc. Phys. 46, 130. Springer, Berlin

Combes F., 1991, ARA\&A 29, 195

Comins N., 1981, MNRAS 194, 169

Comins N.F., 1983, ApJ 266, 543

Comins N.F., 1984, ApJ 284, 90

Comins N.F., Shore S.N., 1990, A\&A 237, 345

Cowie L.L., Rybicki G.B., 1982, ApJ 260, 504

Cross M.C., Hohenberg P.C., 1993, Rev. Modern Phys. 65, 851

de Zeeuw T., Franx M., 1991, ARA\&A 29, 239

Elmegreen B.G., Lada C.J., 1977, ApJ 214, 725

Elmegreen D.M., 1981, ApJS 47, 229

Elmegreen D.M., Elmegreen B.G., 1982, MNRAS 201, 1021

Elmegreen D.M., Elmegreen B.G., 1983, ApJS 54, 127

Feitzinger J.V., 1985, in: The Milky Way Galaxy, IAU Symposium 106. In: van Woerden H., Allen R.J., Burton W.B. (eds.). Reidel, Dordrecht, p. 559

Franco J., Shore S.N., 1984, ApJ 285, 813

Gerola H., Seiden P.E., 1978, ApJ 223, 129

Gerola H., Seiden P.E.,Schulman L.S., 1980, ApJ 242, 517

Glansdorff P., Prigogine I., 1971, Thermodynamic Theory of Structure, Stability and Fluctuations. Wiley, London (XIV and XV)

Goldreich P., Lynden-Bell D., 1965, MNRAS 130, 125

Haken H., 1977, Synergetics. Springer, Berlin 
Heisenberg W., von Weizsäcker C.F., 1948, Zeitschrift für Physik 125, 290

Herrmann H.J., 1983, J. Phys. A16, L611

Hockney R.W., Hohl F., 1969, AJ 74, 1102

Hohl F., 1971, ApJ 168, 343

Hron J., Maitzen H.M., 1985, in: The Milky Way Galaxy, IAU Symposium 106. In: van Woerden H., Allen R.J., Burton W.B. (eds.). Reidel, Dordrecht, p. 105

Ikeuchi S., Yoshioka S., 1989, Comm. Astrophys. 13, 117

Kalnajs A.J., 1972, Astrophys. Lett. 11, 41

Kaufman M., 1979, ApJ 232, 707 and 717

King I.R., 1989, in: The Milky Way as a Galaxy, 19th Advanced Course of the Swiss Society of Astrophysics and Astronomy, Saas-Fee. In: Buser R., King I. (eds.), Geneva Observatory, Sauverny, p. 63

King I.R., 1989a, in: The Milky Way as a Galaxy, 19th Advanced Course of the Swiss Society of Astrophysics and Astronomy, Saas-Fee. In: Buser R., King I. (eds.), Geneva Observatory, Sauverny, p. 161

Lavallée P., Boon J.P., Noullez A., 1993, in: Cellular Automata: Prospects in Astrophysical Applications. In: Perdang J.M., Lejeune A. (eds.), Scientific, Singapore, p. 119

Lin C.C., 1966, Lect. Appl. Math. 9 (Part 2) 66

Lejeune A., 1993, in: Cellular Automata: Prospects in Astrophysical Applications. In: Perdang J.M., Lejeune A. (eds.), World Scientific, Singapore, p. 323

Lejeune A., Perdang J., 1991, in: Computational Physics. In: Tenner A. (ed.), World Scientific, Singapore, p. 407

Lejeune A., Perdang J., 1996 (Paper II)

Lin C.C., Shu F.H., 1964, ApJ 140, 646

Lin C.C., Shu F.H., 1966, Proc. Nation. Acad. Sci. 55, 229

Miller R.H., Prendergast K.H., 1968, ApJ 151, 699

Mueller M.W., Arnett W.D., 1976, ApJ 210, 670

Patsis P.A., Contopoulos G., Grosbøl P., 1991, A\&A 243, 373

Perdang J., 1974, Contact Group in Astronomy and Astrophysics, 19 October, 1974, Leuven, Belgium, FNRS

Perdang J., 1990, Vistas Astron. 33, 249

Perdang J., 1993, in: Cellular Automata: Prospects in Astrophysical Applications. In: Perdang J.M., Lejeune A. (eds.), World Scientific, Singapore, p. 3

Perdang J., 1994, Astrophys. Space Sci. 220, 1

Perdang J., 1995 (in preparation)

Pfenniger D., Friedli D., 1991, A\&A 252, 75

Prigogine I., Lefever R., 1968, J. Chem. Phys. 48, 1695

Raha N., Sellwood J.A., James R.A., Kahn F.D., 1991, Nat 352,411

Rouet J.L., Jamin E., Feix M.R., 1991, in: Applying Fractals in Astrophysics, Springer Lecture Notes in Physics m3. Springer, Berlin, p. 161
Sancisi R., 1973, in: Galactic Radio Astronomy, IAU Symposium 60. In: Kerr F.J., Simonson S.C. (eds.). Reidel, Dordrecht, p. 115

Sandage A., Tammann G.A., 1981, A Revised Shapley-Ames Catalog of Bright Galaxies, Carnegie Institute of Washington Publication 635, Washington D.C. (quoted as RSAC)

Sandage A., Bedke J., 1988, Atlas of Galaxies, NASA SP-496, Washington D.C. (quoted as AG)

Schulman L.S., Seiden P.E., 1983, Ann. Israel Phys. Soc. 5. 251

Schulman L.S., Seiden P.E., 1986, Sci 233, 425

Schulman L.S., 1993, in: Cellular Automata: Prospects in Astrophysical Applications. In: Perdang J.M., Lejeune A. (eds.), World Scientific, Singapore, p. 294

Schwarzschild M., 1979, ApJ 232, 236

Schwarzschild M., 1982, ApJ 263, 599

Seiden P.E., 1985, in: The Milky Way Galaxy, IAU Symposium 106. In: van Woerden H., Allen R.J., Burton W.B. (eds.). Reidel, Dordrecht, p. 551

Seiden P.E., Gerola H., 1979, ApJ 233, 56

Seiden P.E., Gerola H., 1982, Fundament. Cosmic Phys. 7, 241

Seiden P.E., Schulman L.S., 1990, Adv. Phys. 39, 1

Seiden P.E., Schulman L.S., Gerola H., 1979, ApJ 232, 702

Seiden P.E., Schulman L.S., Feitzinger J.V. , 1982, ApJ 253, 91

Sellwood J.A., Carlberg R.G., 1984, ApJ 282, 61

Sellwood J.A., Wilkinson A., 1993, Reports Progress Phys. 56, 173

Sempere M.J., Garca-Burillo S., Combes F., Knapen J.H., 1995, A\&A 296, 45

Shore S.N., 1981, ApJ 249, 93

Shore S.N., 1982, ApJ 265, 202

Shore S.N., 1985, in: Birth and Infancy of Stars, Les Houches, Session 16. In: Lucas R., Omont A., Stora R. (eds.). Elsevier B.V., p. 449

Shore S.N., Ferrini F., Palla F., 1987, ApJ 316, 663

Shu F.H. , 1985, in: The Milky Way Galaxy, IAU Symposium 106. In: van Woerden H., Allen R.J., Burton W.B. (eds.). Reidel, Dordrecht, p. 561

Shu F.H., 1991, The Physics of Astrophysics Vol. II. Gas Dynamics. University Science Books, Mill Valley

Statler T., Comins N., Smith B.F., 1983, ApJ 270, 79

Stauffer D., 1985, Introduction to Percolation Theory, Taylor and Francis, London

van der Kruit P.C., 1989, in: The Milky Way as a Galaxy, 19th Advanced Course of the Swiss Society of Astrophysics and Astronomy, Saas-Fee. In: Buser R., King I. (eds.), Geneva Observatory, Sauverny, p. 185

Young J.S., Scoville N.Z., 1991, ARA\&A 29, 581 